\title{
Low Complexity MLSE Equalization in Highly Dispersive Rayleigh Fading Channels
}

\author{
H. C. Myburgh ${ }^{1}$ and J. C. Olivier ${ }^{1,2}$ \\ ${ }^{1}$ Department of Electrical, Electronic and Computer Engineering, University of Pretoria, Lynnwood Road, 0002 Pretoria, South Africa \\ ${ }^{2}$ Defence Research Unit, CSIR, Meiring Naude Road, 0184 Pretoria, South Africa
}

Correspondence should be addressed to H. C. Myburgh, herman.myburgh@gmail.com

Received 1 October 2009; Revised 29 March 2010; Accepted 30 June 2010

Academic Editor: Xiaoli Ma

Copyright $\odot 2010$ H. C. Myburgh and J. C. Olivier. This is an open access article distributed under the Creative Commons Attribution License, which permits unrestricted use, distribution, and reproduction in any medium, provided the original work is properly cited.

\begin{abstract}
A soft output low complexity maximum likelihood sequence estimation (MLSE) equalizer is proposed to equalize M-QAM signals in systems with extremely long memory. The computational complexity of the proposed equalizer is quadratic in the data block length and approximately independent of the channel memory length, due to high parallelism of its underlying Hopfield neural network structure. The superior complexity of the proposed equalizer allows it to equalize signals with hundreds of memory elements at a fraction of the computational cost of conventional optimal equalizer, which has complexity linear in the data block length but exponential in die channel memory length. The proposed equalizer is evaluated in extremely long sparse and dense Rayleigh fading channels for uncoded BPSK and 16-QAM-modulated systems and remarkable performance gains are achieved.
\end{abstract}

\section{Introduction}

Multipath propagation in wireless communication systems is a challenge that has enjoyed much attention over the last few decades. This phenomenon, caused by the arrival of multiple delayed copies of the transmitted signal at the receiver, results in intersymbol interference (ISI), severely distorting the transmitted signal at the receiver.

Channel equalization is necessary in the receiver to mitigate the effect of ISI, in order to produce reliable estimates of the transmitted information. In the early 1970s, Forney proposed an optimal equalizer [1] based on the Viterbi algorithm (VA) [2], able to optimally estimate the most likely sequence of transmitted symbols. The VA was proposed a few years before for the optimal decoding of convolutional error-correction codes. Shortly afterward, the BCJR algorithm [3], also known as the maximum a posterior probability (MAP) algorithm, was proposed, able to produce optimal estimates of the transmitted symbols.

The development of an optimal MLSE equalizer was an extraordinary achievement, as it enabled wireless communication system designers to design receivers that can optimally detect a sequence of transmitted symbols, corrupted by ISI, for the first time. Although the Viterbi MLSE algorithm and the MAP algorithm estimate the transmitted information with maximum confidence, their computational complexities are prohibitive, increasing exponentially with an increase in channel memory [4]. Their complexity is $O\left(N M^{L-1}\right)$, where $N$ is the data block length, $L$ is the channel impulse response (CIR) length and $M$ is the modulation alphabet size. Due to the complexity of optimal equalizer, they are rendered infeasible in communication systems with moderate to large bandwidth. For this reason, communication system designers are forced to use suboptimal equalization algorithms to alleviate the computational strain of optimal equalization algorithms, sacrificing system performance.

A number of suboptimal equalization algorithms have been considered where optimal equalizers cannot be used due to constrains on the processing power. Although these equalizers allow for decreased computational complexity, their performance is not comparable to that of optimal equalizers. The minimum mean squared error (MMSE) equalizer and the decision feedback equalizer (DFE) [5-7], and variants thereof, are often used in systems where the channel memory is too long for optimal equalizers to be applied $[4,8]$. Orthogonal frequency division multiplexing 
(OFDM) modulation can be used to completely eliminate the effect of multipath on the system performance by exploiting the orthogonality properties of the Fourier matrix and through the use of a cyclic prefix, while maintaining trivial per symbol complexity. OFDM, however, is very susceptible to Doppler shift, suffers from a large peak-toaverage power ratio (PAPR), and requires large overhead when the channel delay spread is very long compared to the symbol period $[4,9]$.

There are a number of communication channels that have extremely long memory. Among these are underwater channels (UAC), magnetic recording channels (MRC), power line channels (PLC), and microwave channels (MWC) [10-13]. In these channels, there may be hundreds of multipath components, leading to severe ISI. Due to the large amount of interfering symbols in these channels, the use of conventional optimal equalization algorithms are infeasible.

In this paper, a low complexity MLSE equalizer, first presented by the authors in [14], (in this paper, the M-QAM HNN MLSE equalizer in [14] is presented in much greater detail. Here, a complete complexity analysis, as well as the performance of the proposed equalizer in sparse channels, are presented) is developed for equalization in M-QAMmodulated systems with extremely long memory. Using the Hopfield neural network (HNN) [15] as foundation, this equalizer has complexity quadratic in the data block length and approximately independent of the channel memory length for practical systems. (In practical systems, the data block length is larger that the channel memory length.) Its complexity is roughly $O\left(4 Z N^{2}+6 L^{2}\right)$, where $Z$ is the number of iterations performed during equalization and $N$ and $L$ are the data block length and CIR length as before. (A complete computational complexity analysis is presented in Section 5) Its superior computational complexity, compared to that of the Viterbi MLSE and MAP algorithms, is due to the high parallelism and high level of interconnection between the neurons of its underlying HNN structure.

This equalizer, henceforth referred to as the HNN MLSE equalizer, iteratively mitigates the effect of ISI, producing near-optimal estimates of the transmitted symbols. The proposed equalizer is evaluated for uncoded BPSK and 16-QAM modulated single-carrier mobile systems with extremely long memory-for (CIRs) of multiple hundredswhere its performance is compared to that of an MMSE equalizer for BPSK modulation. Although there currently exist various variants of the MMSE equalizer in the literature [16-21] — some less computationally complex and others more efficient in terms of performance-the conventional MMSE is nevertheless used in this paper as a benchmark since it is well-known and well-studied. It is shown that the performance of the HNN MLSE equalizer approaches unfaded, zero ISI, matched filter performance as the effective time-diversity due to multipath increases. The performance of the proposed equalizer is also evaluated for sparse channels and it is shown that its performance in sparse channels is superior to its performance in equivalent dense, or nonsparse, channels, (equivalent dense channels will be explained in Section 7) with a negligible computational complexity increase.
It was shown by various authors [22-25] that the problem of MLSE can be solved using the HNN. However, none of the authors applied the equalizer model to systems with extremely long memory in mobile fading channels. Also, none of the authors attempted to develop an HNN-based equalizer for higher order signal constellations. (Only BPSK and QPSK modulation were addressed using short length static channels whereas the proposed equalizer is able to equalize M-QAM signals.) The HNN-based MLSE equalizer was neither evaluated for sparse channels in previous work.

This paper is organized as follows. Section 2 discussed the HNN model, followed by a discussion on the basic principles of MLSE equalization in Section 3. In Section 4, the derivation of the proposed M-QAM HNN MLSE equalizer is discussed, followed by a complete computational complexity analysis of the proposed equalizer in Section 5. Simulation results are presented in Section 6, and conclusions are drawn in Section 7.

\section{The Hopfield Neural Network}

The HNN is a recurrent neural network and can be applied to combinatorial optimization and pattern recognition problems, of which the former of interest in this paper. In 1985, Hopfield and Tank showed how neurobiological computations can be modeled with the use of an electronic circuit [15]. This circuit is shown in Figure 1.

By using basic electronic components, they constructed a recurrent neural network and derived the characteristic equations for the network. The set of equations that describe the dynamics of the system is given by [26]

$$
\begin{gathered}
C_{i} \frac{d u_{i}}{d t}=-\frac{u_{i}}{\tau_{i}}+\sum_{j} T_{i j} I_{i}+I_{i}, \\
V_{i}=g\left(u_{i}\right),
\end{gathered}
$$

with $T_{i j}$, the dots, describing the interconnections between the amplifiers, $u_{1}-u_{N}$ the input voltages of the amplifiers, $V_{1}-V_{N}$ the output voltages of the amplifiers, $C_{1}-C_{N}$ the capacitor values, $\rho_{1}-\rho_{N}$ the resistivity values, and $I_{1}-I_{N}$ the bias voltages of each amplifier. Each amplifier represents a neuron. The transfer function of the positive outputs of the amplifiers represents the positive part of the activation function $g(u)$ and the transfer function of the negative outputs represents the negative part of the activation function $g(u)$ (negative outputs are not shown here).

It was shown in [15] that the stable state of this circuit network can be found by minimizing the function

$$
\mathcal{L}=-\frac{1}{2} \sum_{i=1}^{N} \sum_{j=1}^{N} T_{i j} V_{i} V_{j}-\sum_{i=1}^{N} V_{i} I_{i},
$$

provided that $T_{i j}=T_{j i}$ and $T_{i i}=0$, implying that $T$ is symmetric around the diagonal and its diagonal is zero [15]. There are therefore no self-connections. This function is called the energy function or the Lyapunov function which, by definition, is a monotonically decreasing function, ensuring that the system will converge to a stable state [15]. 


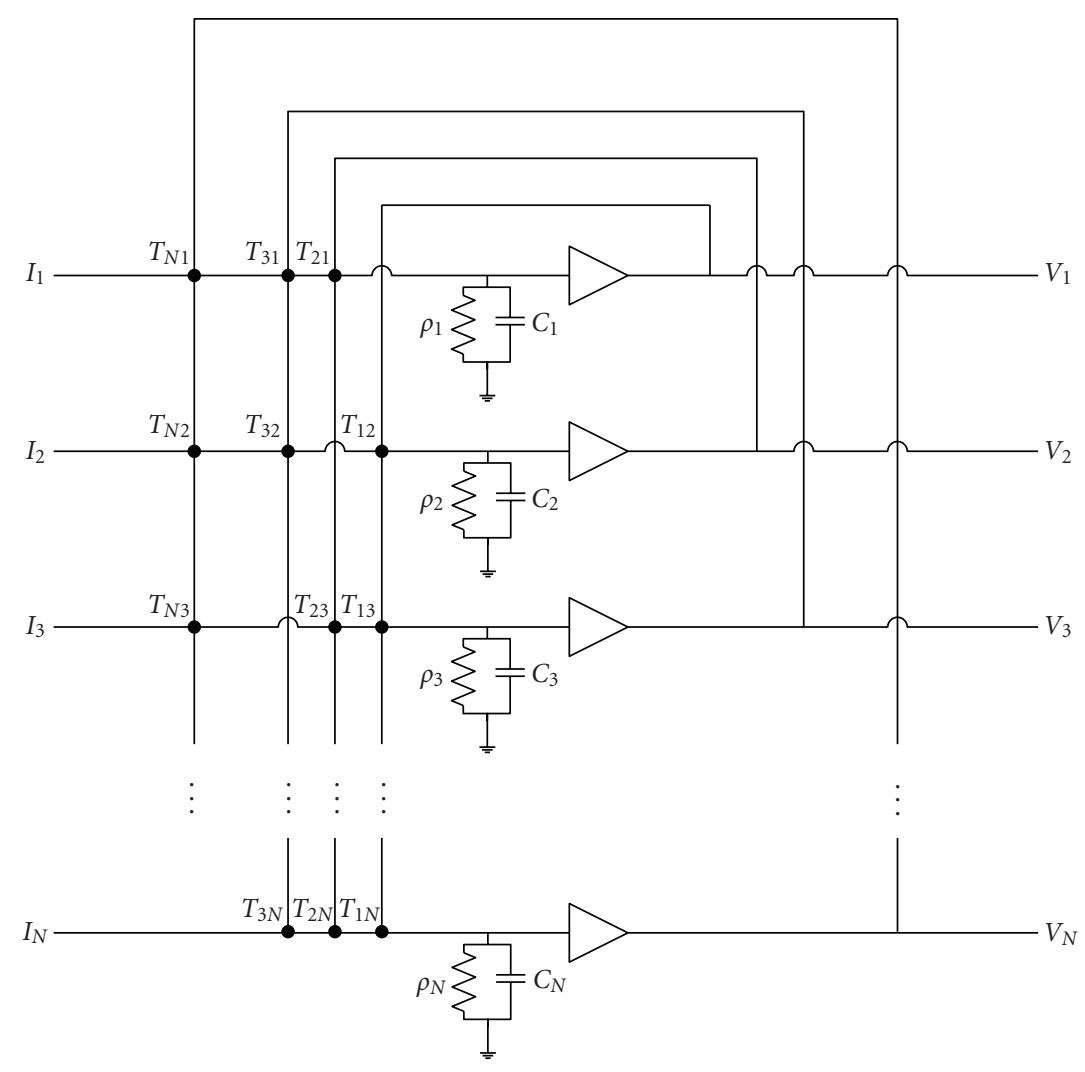

FIgURE 1: Hopfield network circuit diagram.

When minimized, the network converges to a local minimum in the solution space to yield a "good" solution. The solution is not guaranteed to be optimal, but by using optimization techniques, the quality of the solution can be improved. To minimize (2) the system equations in (1) are solved iteratively until the outputs $V_{1}-V_{N}$ settle.

Hopfield also showed that this kind of network can be used to solve the travelling salesman problem (TSP). This problem is of a class called NP-complete, the class of nondeterministic polynomial problems. Problems that fall in this class, can be solved optimally if each possible solution is enumerated [27]. However, complete enumeration is a time-consuming exercise, especially as the solution space grows. Complete enumeration is often not a feasible solution for real-time problems, of which MLSE equalization is considered in this paper.

\section{MLSE Equalization}

In a single-carrier frequency-selective Rayleigh fading environment, assuming a time-invariant channel impulse response (CIR), the received symbols are described by $[1,4]$

$$
r_{k}=\sum_{j=0}^{L-1} h_{j} s_{k-j}+n_{k},
$$

where $s_{k}$ denotes the $k$ th complex symbol in the transmitted sequence of $N$ symbols chosen from an alphabet
$D$ containing $M$ complex symbols, $r_{k}$ is the $k$ th received symbol, $n_{k}$ is the $k$ th Gaussian noise sample $\mathcal{N}\left(0, \sigma^{2}\right)$, and $h_{j}$ is the $j$ th coefficient of the estimated CIR [7]. The equalizer is responsible for reversing the effect of the channel on the transmitted symbols in order to produce the sequence of transmitted symbols with maximum confidence. To optimally estimate the transmitted sequence of length $N$ in a wireless communication system, the cost function [1]

$$
\mathcal{L}=\sum_{k=1}^{N}\left|r_{k}-\sum_{j=0}^{L-1} h_{j} s_{k-j}\right|^{2}
$$

must be minimized. Here, $\mathbf{s}=\left\{s_{1}, s_{2}, \ldots, s_{N}\right\}^{T}$ is the most likely transmitted sequence that will maximize $P(\mathbf{s} \mid \mathbf{r})$. The Viterbi MLSE equalizer is able to solve this problem exactly, with computational complexity linear in $N$ and exponential in $L$ [1]. The HNN MLSE equalizer is also able to minimizes the cost function in (4), with computational complexity quadratic in $N$ but approximately independent of $L$, thus enabling it to perform near-optimal sequence estimation in systems with extremely long CIR lengths at very low computational cost.

\section{The HNN MLSE Equalizer}

It was observed [22-25] that (4) can be written as

$$
\mathcal{L}=-\frac{1}{2} \mathbf{s}^{\dagger} \mathbf{X} \mathbf{s}-\mathbf{I}^{\dagger} \mathbf{s}
$$




\begin{tabular}{|l|l|l|l|l|l|l|l|l|l|l|l|l|l|l|l|l|l|}
\hline$S_{1-L}$ & $S_{2-L}$ & & $S_{0}$ & $S_{1}$ & $S_{2}$ & $S_{3}$ & & & & $S_{N-3}$ & $S_{N-2}$ & $S_{N-1}$ & $S_{N}$ & $S_{N+1}$ & & $S_{N+L-2}$ & $S_{N+L-1}$ \\
\hline
\end{tabular}

(a)

\begin{tabular}{|l|l|l|l|l|l|l|l|l|l|l|l|l|l|}
\hline$r_{1}$ & $r_{2}$ & $r_{3}$ & $r_{4}$ & & $r_{N-4}$ & $r_{N-3}$ & $r_{N-2}$ & $r_{N-1}$ & $r_{N}$ & $r_{N+1}$ & & $r_{N+1-2}$ & $r_{N+L-1}$ \\
\hline
\end{tabular}

(b)

Figure 2: Transmitted (a) and received (b) data block structures. The shaded blocks contain known tail symbols.

where $\mathbf{I}$ is a column vector with $N$ elements, $\mathbf{X}$ is an $N \times N$ matrix, and ${ }^{\dagger}$ implies the Hermitian transpose, where (5) corresponds to the HNN energy function in (2). In order to use the HNN to perform MLSE equalization, the cost function (4) that is minimized by the Viterbi MLSE equalizer must be mapped to the energy function (5) of the HNN. This mapping is performed by expanding (4) for a given block length $N$ and a number of CIR lengths $L$, starting from $L=2$ and increasing $L$ until a definite pattern emerges in $\mathbf{X}$ and $\mathbf{I}$ in (5). The emergence of a pattern in $\mathbf{X}$ and $\mathbf{I}$ enables the realization of an MLSE equalizer for the general case, that is, for systems with any $N$ and $L$, yielding a generalized HNN MLSE equalizer that can be used in a single-carrier communication system.

Assuming that $\mathbf{s}, \mathbf{I}$, and $\mathbf{X}$ contain complex values these variables can be written as [22-25]

$$
\begin{aligned}
\mathbf{s} & =\mathbf{s}_{i}+j \mathbf{s}_{q}, \\
\mathbf{I} & =\mathbf{I}_{i}+j \mathbf{I}_{q}, \\
\mathbf{X} & =\mathbf{X}_{i}+j \mathbf{X}_{q},
\end{aligned}
$$

where $\mathbf{s}$ and $\mathbf{I}$ are column vectors of length $N$, and $\mathbf{X}$ is an $N \times N$ matrix, where subscripts $i$ and $q$ are used to denote the respective in-phase and quadrature components. $\mathbf{X}$ is the cross-correlation matrix of the complex received symbols such that

$$
\mathbf{X}^{H}=\mathbf{X}_{i}^{T}-j \mathbf{X}_{q}^{T}=\mathbf{X}_{i}+j \mathbf{X}_{q},
$$

implying that it is Hermitian. Therefore, $\mathbf{X}_{i}^{T}=\mathbf{X}_{i}$ is symmetric and $\mathbf{X}_{q}^{T}=-\mathbf{X}_{q}$ is skew symmetric [22, 23]. By using the symmetric properties of $\mathbf{X}$, (5) can be expanded and rewritten as

$$
\mathcal{L}=-\frac{1}{2}\left[\mathbf{s}_{i}^{T} \mathbf{X}_{i} \mathbf{s}_{i}+\mathbf{s}_{q}^{T} \mathbf{X}_{q} \mathbf{s}_{q}+2 \mathbf{s}_{q}^{T} \mathbf{X}_{q} \mathbf{s}_{i}\right]-\left[\mathbf{s}_{i}^{T} \mathbf{I}_{i}+\mathbf{s}_{q}^{T} \mathbf{I}_{q}\right],
$$

which in turn can be rewritten as [22-25]

$$
\mathcal{L}=-\frac{1}{2}\left[\mathbf{s}_{i}^{T} \mid \mathbf{s}_{q}^{T}\right]\left[\begin{array}{cc}
\mathbf{X}_{i} & \mathbf{X}_{q}^{T} \\
\mathbf{X}_{q} & \mathbf{X}_{i}
\end{array}\right]\left[\frac{\mathbf{s}_{i}}{\mathbf{s}_{q}}\right]-\left[\mathbf{I}_{i}^{T} \mid \mathbf{I}_{q}^{T}\right]\left[\begin{array}{l}
\mathbf{s}_{i} \\
\mathbf{s}_{q}
\end{array}\right] .
$$

It is clear that (9) is in the form of (5), where the variables in (5) are substituted as follows:

$$
\begin{aligned}
& \mathbf{s}^{\dagger}=\left[\begin{array}{ll}
\mathbf{s}_{i}^{T} \mid & \mathbf{s}_{q}^{T}
\end{array}\right], \\
& \mathbf{I}^{\dagger}=\left[\begin{array}{ll}
\mathbf{I}_{i}^{T} & \mathbf{I}_{q}^{T}
\end{array}\right], \\
& \mathbf{X}=\left[\begin{array}{ll}
\mathbf{X}_{i} & \mathbf{X}_{q}^{T} \\
\mathbf{X}_{q} & \mathbf{X}_{i}
\end{array}\right] .
\end{aligned}
$$

Equation (9) will be used to derive a general model for MQAM equalization.

4.1. Systematic Derivation. The transmitted and received data block structures are shown in Figure 2, where it is assumed that $L-1$ known tail symbols are appended and prepended to the block of payload symbols. (The transmitted tails are $s_{1-L}$ to $s_{0}$ and $s_{N+1}$ to $s_{N+L-1}$ and are equal to $1 / \sqrt{2}+$ $j(1 / \sqrt{2}))$

The expressions for the unknowns in (9) are found by expanding (4), for a fixed data block length $N$ and increasing CIR length $L$ and mapping it to (9). Therefore, for a singlecarrier system with a data block of length $N$ and CIR of length $L$, with the data block initiated and terminated by $L-1$ known tail symbols, $\mathbf{X}_{i}$ and $\mathbf{X}_{q}$ are given by

$$
\mathbf{X}_{i}=-\left[\begin{array}{cccccc}
0 & \alpha_{1} & \cdots & \alpha_{L-1} & \cdots & 0 \\
\alpha_{1} & 0 & \alpha_{1} & \cdots & \ddots & \vdots \\
\vdots & \alpha_{1} & 0 & \ddots & \vdots & \alpha_{L-1} \\
\alpha_{L-1} & \vdots & \ddots & \ddots & \alpha_{1} & \vdots \\
\vdots & \ddots & \cdots & \alpha_{1} & 0 & \alpha_{1} \\
0 & \ddots & \alpha_{L-1} & \cdots & \alpha_{1} & 0
\end{array}\right],
$$

where $\alpha=\left\{\alpha_{1}, \alpha_{2}, \ldots, \alpha_{L-1}\right\}$ and $\gamma=\left\{\gamma_{1}, \gamma_{2}, \ldots, \gamma_{L-1}\right\}$ are respectively determined by

$$
\begin{aligned}
& \alpha_{k}=\sum_{j=0}^{L-k-1} h_{j}^{(i)} h_{j+k}^{(i)}+\sum_{j=0}^{L-k-1} h_{j}^{(q)} h_{j+k}^{(q)}, \\
& \gamma_{k}=\sum_{j=0}^{L-k-1} h_{j}^{(q)} h_{j+k}^{(i)}-\sum_{j=0}^{L-k-1} h_{j}^{(i)} h_{j+k}^{(q)},
\end{aligned}
$$


where $k=1,2,3, \ldots, L-1$ and $i$ and $q$ denote the real and complex components of the CIR coefficients. Also,

$$
\mathbf{I}_{i}=\left[\begin{array}{c}
\lambda_{1}-\rho\left(\alpha_{1}+\gamma_{1}+\cdots+\alpha_{L-1}+\gamma_{L-1}\right) \\
\lambda_{2}-\rho\left(\alpha_{2}+\gamma_{2}+\cdots+\alpha_{L-1}+\gamma_{L-1}\right) \\
\lambda_{3}-\rho\left(\alpha_{3}+\gamma_{3}+\cdots+\alpha_{L-1}+\gamma_{L-1}\right) \\
\vdots \\
\vdots \\
\lambda_{L-1}-\rho\left(\alpha_{L-1}+\gamma_{L-1}\right) \\
\lambda_{L} \\
\vdots \\
\vdots \\
\lambda_{N-L+1} \\
\lambda_{N-L+2}-\rho\left(\alpha_{L-1}-\gamma_{L-1}\right) \\
\vdots \\
\vdots \\
\lambda_{N-2}-\rho\left(\alpha_{3}-\gamma_{3}+\cdots+\alpha_{L-1}-\gamma_{L-1}\right) \\
\lambda_{N-1}-\rho\left(\alpha_{2}-\gamma_{2}+\cdots+\alpha_{L-1}-\gamma_{L-1}\right) \\
\lambda_{N}-\rho\left(\alpha_{1}-\gamma_{1}+\cdots+\alpha_{L-1}-\gamma_{L-1}\right)
\end{array}\right]
$$

where $\rho=1 / \sqrt{2}$ and $\lambda=\left\{\lambda_{1}, \lambda_{2}, \ldots, \lambda_{N}\right\}$ is determined by

$$
\lambda_{k}=\sum_{j=0}^{L-1} r_{j+k}^{(i)} h_{j}^{(i)}+\sum_{j=0}^{L-1} r_{j+k}^{(q)} h_{j}^{(q)},
$$

and $\omega=\left\{\omega_{1}, \omega_{2}, \ldots, \omega_{N}\right\}$ is determined by

$$
\omega_{k}=\sum_{j=0}^{L-1} r_{j+k}^{(q)} h_{j}^{(i)}-\sum_{j=0}^{L-1} r_{j+k}^{(i)} h_{j}^{(q)},
$$

where $k=1,2,3, \ldots, N$ with $i$ and $q$ again denoting the real and complex components of the respective vectors.
4.2. Training. Since the proposed equalizer is based on a neural network, it has to be trained. The HNN MLSE equalizer does not have to be trained by providing a set of training examples as in the case of conventional supervised neural networks [28]. Rather, the HNN MLSE equalizer is trained anew in an unsupervized fashion for each received data block by using the coefficients of the estimated CIR to determine $\alpha_{k}$ in (13) and $\gamma_{k}$ in (14), for $k=1,2,3, \ldots, L-1$, which serve as the connection weights between the neurons. $\mathbf{X}_{i}, \mathbf{X}_{q}, \mathbf{I}_{i}$, and $\mathbf{I}_{q}$ fully describes the structure of the equalizer for each received data block, which are determined according to (11), (12), (15) and (16), using the estimated CIR and the received symbol sequence. $\mathbf{X}_{i}$ and $\mathbf{X}_{q}$ therefore describe the connection weights between the neurons, and $\mathbf{I}_{i}$ and $\mathbf{I}_{q}$ represent the input of the neural network.

4.3. The Iterative System. In order for the HNN to minimize the energy function (5), the following dynamic system is used

$$
\frac{d \mathbf{u}}{d t}=-\frac{\mathbf{u}}{\tau}+\mathbf{T s}+\mathbf{I}
$$

where $\tau$ is an arbitrary constant and $\mathbf{u}=\left\{u_{1}, u_{2}, \ldots, u_{N}\right\}^{T}$ is the internal state of the network. An iterative solution for (19) is given by

$$
\begin{gathered}
\mathbf{u}^{(n)}=\mathbf{T s}^{(n-1)}+\mathbf{I}, \\
\mathbf{s}^{(n)}=g\left(\beta(n) \mathbf{u}^{(n)}\right),
\end{gathered}
$$

where again $\mathbf{u}=\left\{u_{1}, u_{2}, \ldots, u_{N}\right\}^{T}$ is the internal state of the network, $\mathbf{s}=\left\{s_{1}, s_{2}, \ldots, s_{N}\right\}^{T}$ is the vector of estimated symbols, $g(\cdot)$ is the decision function associated with each neuron and $n$ indicates the iteration number. $\beta(\cdot)$ is a function used for optimization.

To determine the MLSE estimate for a data block of length $N$ with $L$ CIR coefficients for a M-QAM system, the following steps are executed:

(1) Use the received symbols $\mathbf{r}$ and the estimated CIR $\mathbf{h}$ to calculate $\mathbf{T}_{i}, \mathbf{T}_{q}, \mathbf{I}_{i}$ and $\mathbf{I}_{q}$ according to (11), (12), (15) and (16).

(2) Initialize all elements in $\left[\mathbf{s}_{i}^{T} \mid \mathbf{s}_{q}^{T}\right]$ to 0 .

(3) Calculate $\left[\mathbf{u}_{i}^{T} \mid \mathbf{u}_{q}^{T}\right]^{(n)}=\left[\begin{array}{ll}\mathbf{X}_{i} & \mathbf{X}_{q}^{T} \\ \mathbf{X}_{q} & \mathbf{X}_{i}\end{array}\right]\left[\mathbf{s}_{i} / \mathbf{s}_{q}\right]^{(n-1)}+\left[\mathbf{I}_{i}^{T} \mid\right.$ $\left.\mathbf{I}_{q}^{T}\right]$.

(4) Calculate $\left[\mathbf{s}_{i}^{T} \mid \mathbf{s}_{q}^{T}\right]^{(n)}=g\left(\beta(n)\left[\mathbf{u}_{i}^{T} \mid \mathbf{u}_{q}^{T}\right]^{(n)}\right)$.

(5) Go to step (2) and repeat until $n=Z$, where $Z$ is the predetermined number of iterations. $(Z=20$ iterations are used for the proposed equalizer.)

As is clear from the algorithm, the estimated symbol vector $\left[\mathbf{s}_{i}^{T} \mid \mathbf{s}_{q}^{T}\right]$ is updated with each iteration. $\left[\mathbf{I}_{i}^{T} \mid \mathbf{I}_{q}^{T}\right]$ contains the best linear estimate for $\mathbf{s}$ (it can be shown that $\left[\mathbf{I}_{i}^{T} \mid\right.$ $\mathbf{I}_{q}^{T}$ ] contains the output of a RAKE reciever used in DSSS systems) and is therefore used as input to the network, while $\left[\begin{array}{ll}\mathbf{X}_{i} & \mathbf{X}_{q}^{T} \\ \mathbf{X}_{q} & \mathbf{X}_{i}\end{array}\right]$ contains the cross-correlation information of 


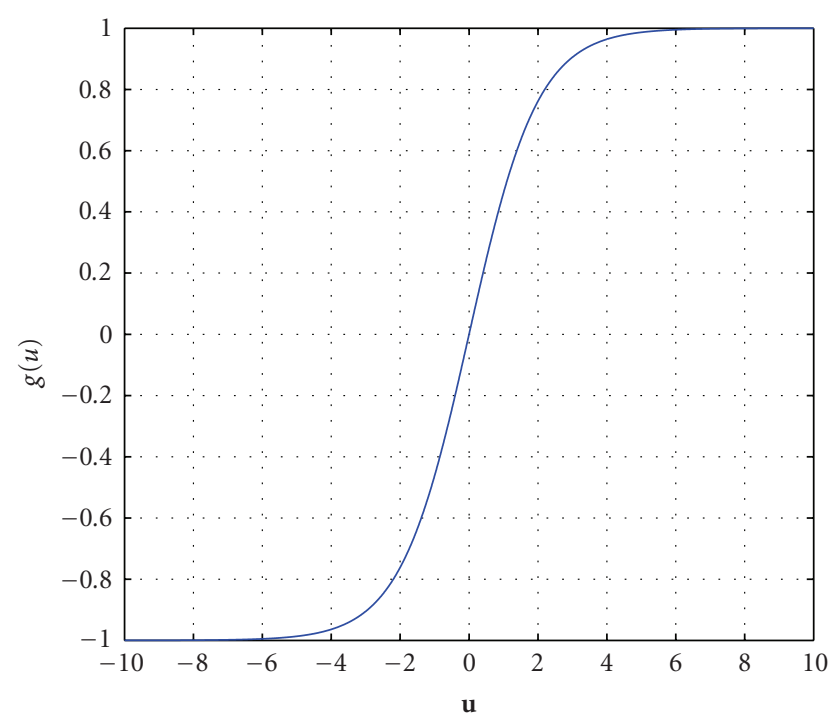

FIGURE 3: The bipolar decision function.

the received symbols. The system solves (4) by iteratively mitigating the effect of ISI and produces the MLSE estimates in $\mathbf{s}$ after $Z$ iterations.

\subsection{The Decision Function}

4.4.1. Bipolar Decision Function. When BPSK modulation is used, only two signal levels are required to transmit information. Therefore, since only two signal levels are used, a bipolar decision function is used in the HNN BPSK MLSE equalizer. This function, also called a bipolar sigmoid function, is expressed as

$$
g(u)=\frac{2}{1+e^{-u}}-1
$$

and is shown in Figure 3. It must also be noted that the bipolar decision can also be used in the M-QAM model for equalization in 4-QAM systems. This is an exception, since, although 4-QAM modulation uses four signal levels, there are only two signal levels per dimension. By using the model derived for M-QAM modulation, 4-QAM equalization can be performed by using the bipolar decision function in (21), with the output scaled by 'n factor $1 / \sqrt{2}$.

4.4.2. Multilevel Decision Function. Apart from 4-QAM modulation, all other M-QAM modulation schemes use multiple amplitude levels to transmit information as the "AM" in the acronym M-QAM implies. A bipolar decision function will therefore not be sufficient; a multilevel decision function with $Q=\log _{2}(M)$ distinct signal levels must be used, where $M$ is the modulation alphabet size.

A multilevel decision function can be realized by adding several bipolar decision functions and shifting each by a predetermined value, and scaling the result accordingly [29, 30]. To realize a $Q$-level decision function for use in an

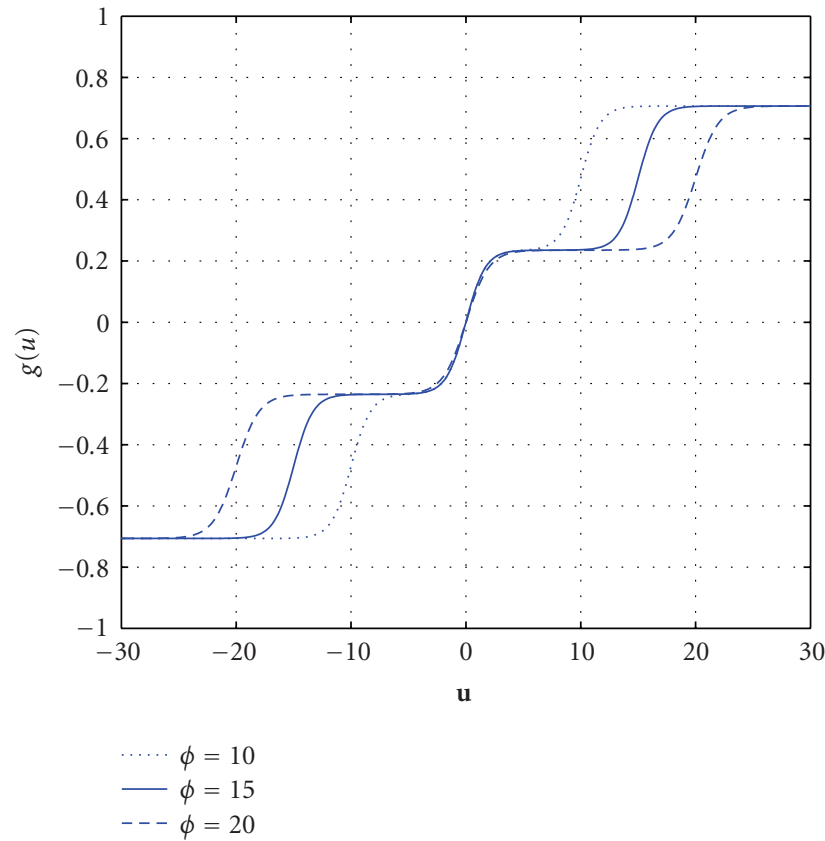

Figure 4: The four-level decision function.

M-QAM HNN MLSE equalizer, the following function can be used:

$$
g(u)=\frac{2}{\sqrt{2}(Q-1)}\left(\sum_{k=-((Q / 2)-1)}^{(Q / 2)-1} \frac{1}{1+e^{-(u+\phi k)}}\right)-\frac{1}{\sqrt{2}},
$$

where $M$ is the modulation alphabet size and $\phi$ is the value by which the respective bipolar decision functions are shifted. Figure 4 shows the four-level decision function used for the 16-QAM HNN MLSE equalizer, for $\phi=10, \phi=15$ and $\phi=$ 20.

Due to the time-varying nature of a mobile wireless communication channel and energy losses caused by absorption and scattering, the total power in the received signal is also time-variant. This complicates equalization when using the M-QAM HNN MLSE equalizer, since the value by which the respective bipolar decision functions are shifted, $\phi$, is dependent on the power in the channel and will therefore have a different value for every new data block arriving at the receiver. For this reason the Q-level decision function in (22) will change slightly for every data block. $\phi$ is determined by the Euclidean norm of the estimated CIR and is given by

$$
\phi=\|\mathbf{h}\|=\sqrt{\left(\sum_{k=0}^{L-1}\left(h_{k}^{(i)}\right)^{2}\right)^{2}+\left(\sum_{k=0}^{L-1}\left(h_{k}^{(q)}\right)^{2}\right)^{2}}
$$

where $h_{k}^{(i)}$ and $h_{k}^{(q)}$ are the $k$ th respective in-phase and quadrature components of the estimated CIR of length $L$ as before.

Figure 4 shows the four-level decision function for different values of $\phi$ to demonstrate the effect of varying power levels in the channel. Higher power in $\mathbf{h}$ will cause the outer neurons to move away from the origin whereas lower 
power will cause the outer neurons to move towards the origin. Therefore, upon reception of a complete data block, $\phi$ is determined according to the power of the CIR, after which equalization commences.

4.5. Optimization. Because MLSE is an NP-complete problem, there are a number of possible "good" solutions in the multidimensional solution space. By enumerating every possible solution, it will be possible to find the best solution, that is, the sequence of symbols that minimizes (4) and (5), but it is not computationally feasible for systems with large $N$ and $L$. The HNN is used to minimize (5) to find a nearoptimal solution at very low computational cost. Because the HNN usually gets stuck in suboptimal local minima, it is necessary to employ optimization techniques as suggested [31]. To aid the HNN in escaping less optimal basins of attraction simulated annealing and asynchronous neuron updates are often used.

Markov Chain Monte Carlo (MCMC) algorithms are used together with Gibbs sampling in [32] to aid optimization in the solution space. According to [32], however, the complexity of the MCMC algorithms may become prohibitive due to the so called stalling problem, which result from low probability transitions in the Gibbs sampler. To remedy this problem an optimization variable referred to as the "temperature" can be adjusted in order to avoid these small transition probabilities. This idea is similar to simulated annealing, where the temperature is adjusted to control the rate of convergence of the algorithm as well as the quality of the solution it produces.

4.5.1. Simulated Annealing. Simulated annealing has its origin in metallurgy. In metallurgy annealing is the process used to temper steel and glass by heating them to a high temperature and then gradually cooling them, thus allowing the material to coalesce into a low-energy crystalline state [28]. In neural networks, this process is imitated to ensure that the neural network escapes less optimal local minima to converge to a near-optimal solution in the solution space. As the neural network starts to iterate, there are many candidate solutions in the solution space, but because the neural network starts to iterate at a high temperature, it is able to escape the less optimal local minima in the solutions space. As the temperature decreases, the network can still escape less optimal local minima, but it will start to gradually converge to the global minimum in the solution space to minimize the energy. This state of minimum energy corresponds to the optimal solution.

The output of the function $\beta(\cdot)$ in (20) is used for simulated annealing. As the system iterates, $n$ is incremented with each iteration, and $\beta(\cdot)$ produces a value according to an exponential function to ensure that the system converges to a near-optimal local minimum in the solution space. This function is give by

$$
\beta(n-1)=5^{2(n-Z+1) / Z},
$$

and shown in Figure 5 . This causes the output of $\beta(\cdot)$ to start at a near-zero value and to exponentially converge to 1 with each iteration.

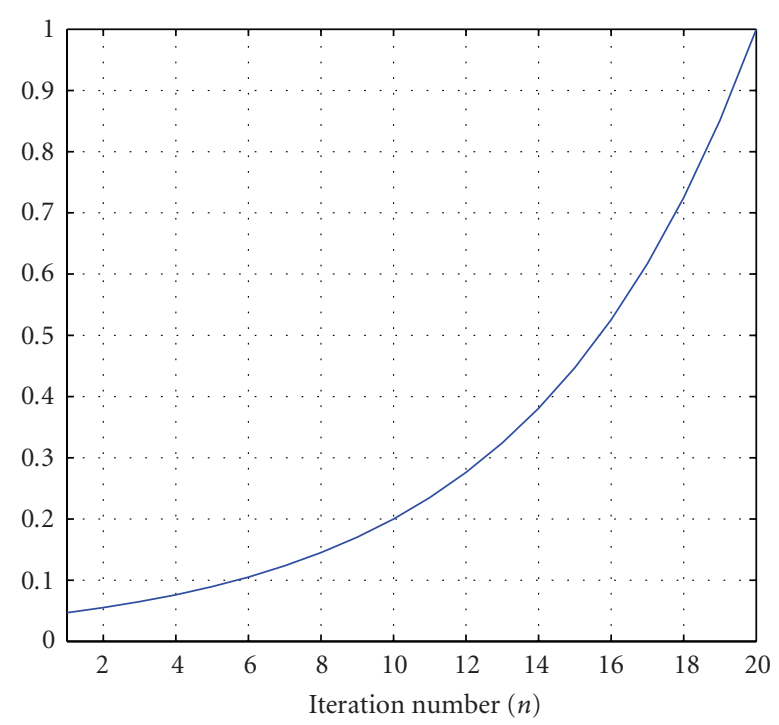

Figure 5: $\beta$-updates for $Z=20$ iterations.

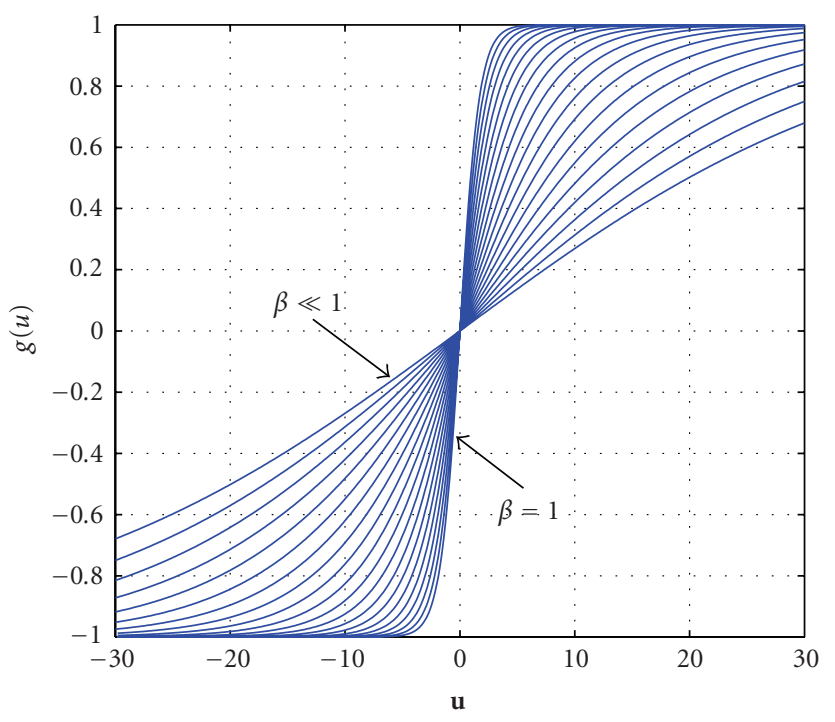

FIGURE 6: Simulated annealing on the bipolar decision function for $Z=20$ iterations.

The effect of annealing on the bipolar and four-level decision function during the iteration cycle is shown in Figures 6 and 7, respectively, with the slope of the decision function increasing as $\beta(\cdot)$ is updated with each iteration. Simulated annealing ensures near-optimal sequence estimation by allowing the system to escape less optimal local minima in the solution space, leading to better system performance.

Figures 8 and 9 show the neuron outputs, for the real and complex symbol components, of the 16-QAM HNN MLSE equalizer for each iteration of the system with and without annealing. It is clear that annealing allows the outputs of the neurons to gradually evolve in order to converge to nearoptimal values in the $\mathrm{N}$-dimensional solution space, not produce reliable transmitted symbol estimates. 


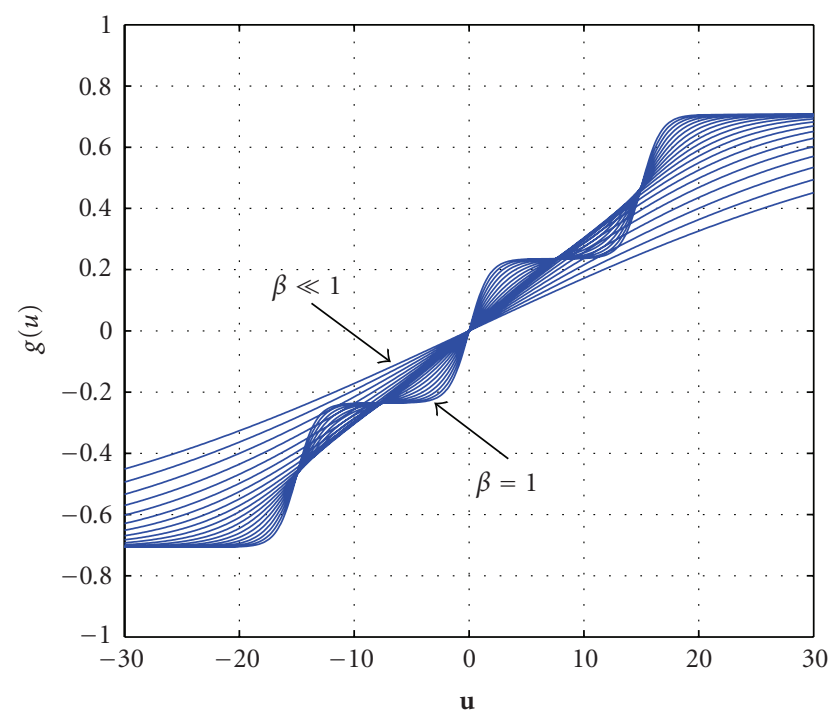

FIGURE 7: Simulated annealing on the four-level decision function for $Z=20$ iterations.

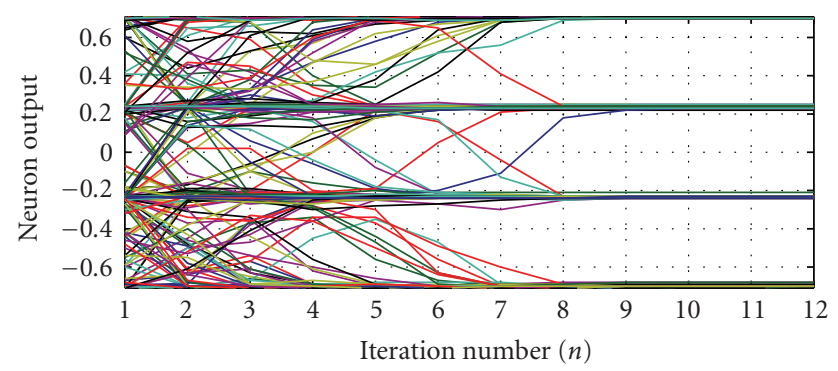

FIgURE 8: Convergence of the 16-QAM HNN MLSE equalizer without annealing.

4.5.2. Asynchronous Updates. In artificial neural networks, the neurons in the network can either be updated using parallel or asynchronous updates. Consider the iterative solution of the HNN in (20). Assume that $\mathbf{u}, \mathbf{s}$ and I each contain $N$ elements and that $\mathrm{T}$ is an $N \times N$ matrix with $Z$ iterations as before.

When parallel neuron updates are used, $N$ elements in $\mathbf{u}^{(n)}$ are calculated before $N$ elements in $\mathbf{s}^{(n)}$ are determined, for each iteration. This implies that the output of the neurons will only be a function of the neuron outputs from the previous iteration. On the other hand, when using asynchronous neuron updates, one element in $\mathbf{s}^{(n)}$ is determined for every corresponding element in $\mathbf{u}^{(n)}$. This is performed $N$ times per iteration-once for each neuron. Asynchronous updates allow the changes of the neuron outputs to propagate to the other neurons immediately [31], while the output of all of the $N$ neurons will only be propagated to the other neurons after all of them have been updated when parallel updates are used.

With parallel updates the effect of the updates propagates through the network only after one complete iteration cycle. This implies that the energy of the network might change

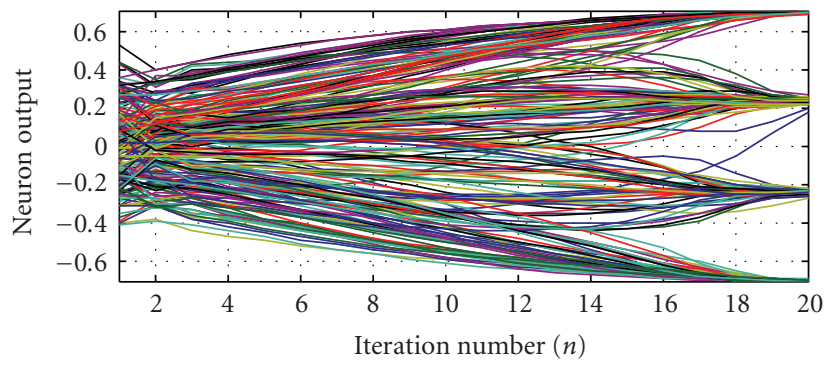

FIGURE 9: Convergence of the 16-QAM HNN MLSE equalizer with annealing.

drastically, because all of the neurons are updated together. This will cause the state of the neural network to "jump" around on the solution space, due to the abrupt changes in the internal state of the network. This will lead to degraded performance, since the network is not allowed to gradually evolve towards an optimal, or at least a near-optimal, basin of attraction.

With asynchronous updates the state of the network changes after each element in $\mathbf{u}^{(n)}$ is determined. This means that the state of the network undergoes $N$ gradual changes during each iteration. This ensures that the network traverses the solution space using small steps while searching for the global minimum. The computational complexity is identical for both parallel and asynchronous updates [31]. Asynchronous updates are therefore used for the HNN MLSE equalizer. The neurons are updated in a sequential order: $1,2,3, \ldots, N$.

4.6. Convergence and Performance. The rate of convergence and the performance of the HNN MLSE equalizer are dependent on the number of CIR coefficients $L$ as well as the number of iterations $Z$. Firstly, the number of CIR coefficients determines the level of interconnection between the neurons in the network. A long CIR will lead to dense population of the connection matrix in $\mathbf{X}(10)$, consisting of $\mathbf{X}_{i}$ in (11) and $\mathbf{X}_{q}$ (12), which translates to a high level of interconnection between the neurons in the network. This will enable the HNN MLSE equalizer to converge faster while producing better maximum likelihood sequence estimates, which is ultimately the result of a high level of diversity provided by a highly dispersive channel. Similarly, a short CIR will result in a sparse connection matrix $\mathbf{X}$, where the HNN MLSE equalizer will converge slower while yielding less optimal maximum likelihood sequence estimates.

Second, simulated annealing, which allows the neuron outputs to be forced to discrete decision levels when the iteration number reaches the end of the iteration cycle (when $n=Z$ ), ensures that the HNN MLSE equalizer will have converged by the last iteration (as dictated by $Z$ ). This is clear from Figure 9. For small $Z$, the output of the HNN MLSE equalizer will be less optimal than for large $Z$. It was found that the HNN MLSE equalizer produces acceptable performance without excessive computational complexity for $Z=20$. 
4.7. Soft Outputs. To enable the HNN MLSE equalizer to produce soft outputs, $\beta(\cdot)$ in (24) is scaled by a factor 0.5 . This allows the outputs of the equalizer to settle between the discrete decision levels instead of being forced to settle on the decision levels.

\section{Computational Complexity Analysis}

The computational complexity of the HNN MLSE equalizer is quadratic in the data block length $N$ and approximately independent of the CIR length $L$ for practical systems where the data block length is larger than channel memory length. This is due to the high parallelism of its underlying neural network structure an high level of interconnection between the neurons. The approximate independence of the complexity from the channel memory is significant, as the CIR length is the dominant term in the complexity of all optimal equalizers, where the complexity is $O\left(N M^{L-1}\right)$.

In this section, the computational complexity of the HNN MLSE equalizer is analyzed, where it is compared to that of the Viterbi MLSE equalizer. The computational complexities of these algorithms are analyzed by assuming that an addition as well as a multiplication are performed using one machine instruction. It is also assumed that variable initialization does not add to the cost.

5.1. HNN MLSE Equalizer. The M-QAM HNN MLSE equalizer performs the following steps. (The computational complexity of the BPSK HNN MLSE equalizer is easily derived from that of the M-QAM HNN MLSE equalizer.)

(1) Determine $\alpha$ and $\gamma$ values using the estimated CIR: There are $L-1$ distinct $\alpha$ values and $L-1$ distinct $\gamma$ values. $\alpha_{1}$ and $\gamma_{1}$ both contain $L-1$ terms, each consisting of a multiplication between two values. Also, $\alpha_{L-1}$ and $\gamma_{L-1}$ both contain one term. Therefore the number of computations to determine all $\alpha$ - and $\gamma$ values can be written as

$$
\begin{aligned}
2 \sum_{n=1}^{L-1} n+2 \sum_{n=1}^{L-1} n= & 4 \sum_{n=1}^{L-1} n \\
= & 4(1+2+\cdots+(L-1)) \\
= & 4(((\mathrm{~L}-1)+1)+((\mathrm{L}-2)+2) \\
& +((\mathrm{L}-3)+3)+((\mathrm{L}-(\mathrm{L}+1)) \\
& +(L+1))) \\
= & 4(L-1)\left(\frac{L-1}{2}\right) \\
= & 2(L-1)^{2} .
\end{aligned}
$$

(2) Populate matrices $\mathbf{T}_{i}$ and $\mathbf{T}_{q}$ (of size $N \times N$ ) and vectors $\mathbf{I}_{i}$ and $\mathbf{I}_{q}$ (of size $N$ ). Under the assumption that variable initialization does not add to the total cost, the population of $\mathbf{T}_{i}$ and $\mathbf{T}_{q}$ does not add to the cost. However, $\mathbf{I}_{i}$ and $\mathbf{I}_{q}$ are not only populated, but some calculations are performed before population. All elements in $\mathbf{I}_{i}$ and $\mathbf{I}_{q}$ need $L$ additions of two multiplicative terms. Also, the first and the last $L-1$ elements in $\mathbf{I}_{i}$ and $\mathbf{I}_{q}$ together contain $(L-1)^{2} \alpha$ and $\gamma$ addition and/or subtraction terms. Therefore, the cost of populating $\mathbf{I}_{i}$ and $\mathbf{I}_{q}$ is given by

$$
\sum_{n=1}^{2 N} \sum_{m=1}^{L-1} m+4(L-1)^{2}=2 N(L-1)+4(L-1)^{2} .
$$

(3) Initialize $\mathbf{s}=\left[\mathbf{s}_{i}^{T} \mid \mathbf{s}_{q}^{T}\right]$ and $\mathbf{u}=\left[\mathbf{u}_{i}^{T} \mid \mathbf{u}_{q}^{T}\right]$, both of length $2 N$. Under the assumption that variable initialization does not add to the total cost, initialization of these variables does not add to the cost.

(4) Iterate the system $Z$ times:

(i) Determine the state vector $\left[\begin{array}{lll}\mathbf{u}_{i}^{T} & \mid & \mathbf{u}_{q}^{T}\end{array}\right]^{(n)}=$ $\left[\begin{array}{ll}\mathbf{X}_{i} & \mathbf{X}_{q}^{T} \\ \mathbf{X}_{q} & \mathbf{X}_{i}\end{array}\right]\left[\mathbf{s}_{i} / \mathbf{s}_{q}\right]^{(n-1)}+\left[\mathbf{I}_{i}^{T} \mid \mathbf{I}_{q}^{T}\right]$. The cost of multiplying a matrix of size $2 \mathrm{~N} \times 2 \mathrm{~N}$ with a vector of size $2 \mathrm{~N}$ and adding another vector of length $2 \mathrm{~N}$ to the result, $Z$ times, is given by

$$
\begin{aligned}
Z\left(\sum_{n=1}^{2 N}\left(\sum_{m=1}^{2 N} m\right)+2 N\right) & =Z\left((2 N)^{2}+2 N\right) \\
& =(2 N)^{2} Z+2 Z N
\end{aligned}
$$

(ii) Calculate $\left[\begin{array}{lll}\mathbf{s}_{i}^{T} & \mid & \mathbf{s}_{q}^{T}\end{array}\right]^{(n)}=g\left(\beta(n)\left[\begin{array}{lll}\mathbf{u}_{i}^{T} & \mid \mathbf{u}_{q}^{T}\end{array}\right]^{(n-1)}\right) Z$ times. The cost of calculating the estimation vector $\mathbf{s}$ of length $2 N$ by using every value in state vector $\mathbf{u}$, also of length $2 N$, assuming that the sigmoid function uses three instructions to execute, $Z$ times, is given by (it is assumed that the values of $\beta(n)$ is stored in a lookup table, where $n=1,2,3, \ldots, Z$, to trivialize the computational complexity of simulated annealing)

$$
3 \times 2 Z N=6 Z N .
$$

Thus, by adding all of the computations above, the total computational complexity for the M-QAM HNN MLSE equalizer is

$$
(2 N)^{2} Z+8 Z N+2 N(L-1)+6(L-1)^{2} .
$$

The structure of the M-QAM HNN MLSE equalizer is identical to that of the BPSK HNN MLSE equalizer. The only difference is that, for the BPSK HNN MLSE equalizer, all matrices and vectors are of dimension $N$ and instead of $2 N$, as only the in-phase component of the estimated symbol vector is considered. Therefore, it follows that the computational complexity of the BPSK HNN MLSE equalizer will be

$$
N^{2} Z+4 Z N+N(L-1)+3(L-1)^{2} .
$$

5.2. Viterbi MLSE Equalizer. The Viterbi equalizer performs the following steps:

(1) Setup trellis of length $N$, where each stage contains $M^{L-1}$ states, where $M$ is the constellation size: It is assumed that this does not add to the complexity. It must however be noted that the dimensions of the trellis is $N \times M^{L-1}$. 
(2) Determine two Euclidean distances for each node. The Euclidean distance is determined by subtracting $L$ addition terms, each containing one multiplication, from the received symbol at instant $k$. The cost for determining one Euclidean distance is therefore given by

$$
2 N M^{L-1}(2 L+1)=4 L N M^{L-1}+2 N M^{L-1} .
$$

(3) Eliminate contending paths at each node. Two path costs are compared using an if-statement, assumed to count one instruction. The cost is therefore

$$
2 N M^{L-1} \text {. }
$$

(4) Backtrack the trellis to determine the MLSE solution. Backtracking accross the trellis, where each time instant $k$ requires an if-statement. The cost is therefore

$$
N M^{L-1}
$$

Adding all costs to give the total cost results in

$$
4 L N M^{L-1}+5 N M^{L-1} \text {. }
$$

\subsection{HNN MLSE Equalizer and Viterbi MLSE Equalizer Com-} parison. Figure 10 shows the computational complexities of the HNN MLSE equalizer and the Viterbi MLSE equalizer as a function of the CIR length $L$, for $L=2$ to $L=10$, where the number of iterations is $Z=20$. For the HNN MLSE equalizer, it is shown for BPSK and M-QAM modulation, since the computational complexities of all M-QAM HNN MLSE equalizers are equal. Also, for the Viterbi MLSE equalizer, it is shown for BPSK and 4-QAM modulation. It is clear that the computational complexity of the HNN MLSE equalizer is superior to that of the Viterbi MLSE equalizer for system with larger memory. For BPSK, the break-even mark between the HNN MLSE equalizer and the Viterbi MLSE equalizer is at $L=7$, and for 4 -QAM it is at $L=4.2 \approx 4$.

The complexity of the HNN MLSE equalizer for both BPSK and M-QAM seems constant whereas that of the Viterbi MLSE equalizer increases exponentially as the CIR length increases. Also, note the difference in complexity between the BPSK HNN MLSE equalizer and the MQAM HNN MLSE equalizer. This is due to the quadratic relationship between the complexity and the data block length, which dictates the size of the vectors and matrices in the HNN MLSE equalizer. The HNN MLSE equalizer is however not well-suited for systems with short CIRs, as the complexity of the Viterbi MLSE equalizer is less than that of the HNN MLSE equalizer for short CIRs. This is however not a concern, since the aim of the proposed equalizer is on equalization of signals in systems with extremely long memory.

Figure 11 shows the computational complexity of the HNN MLSE equalizer for BPSK and M-QAM modulation for block lengths of $N=100$ and $N=500$, respectively, indicating the quadratic relationship between the computational complexity and the data block length, also requiring

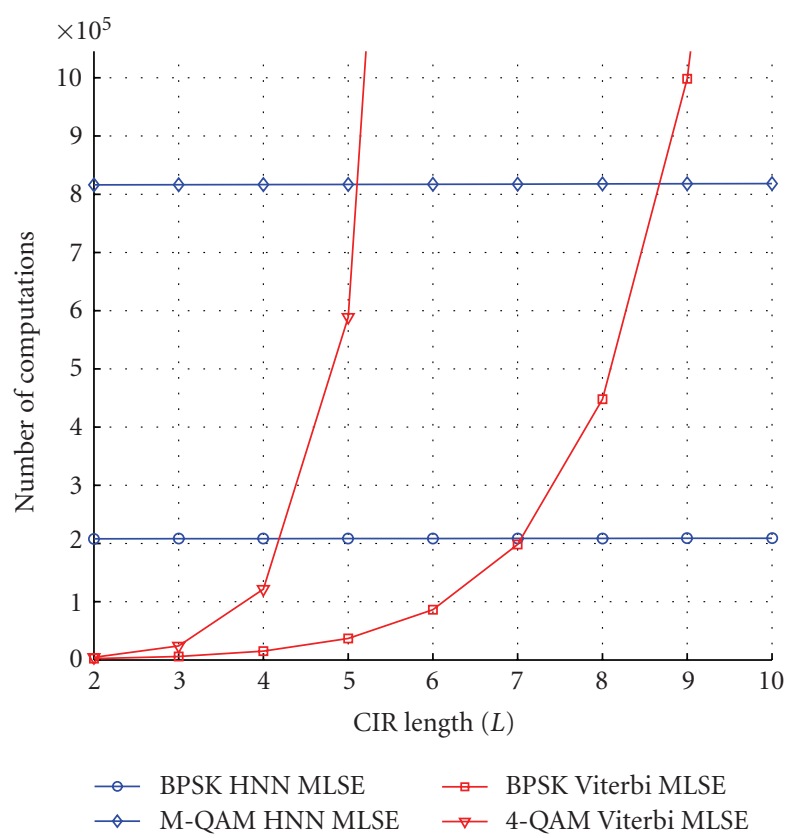

FIGURE 10: Computational complexity comparison between the HNN MLSE and Viterib MLSE equalizers.

larger vectors and matrices in the HNN MLSE equalizer. It is clear that, as the data block length increases, the data block length $N$, rather than the CIR length $L$, is the dominant factor contributing to the computational complexity. However, due to the approximate independence of the complexity on the CIR length, the HNN MLSE equalizer is able to equalize signals in systems with hundreds of CIR elements for large data block lengths. This should be clear from Figures 10 and 11.

The scenario in Figure 11 is somewhat unrealistic, since the data block length must at least be as long as the CIR length. However, Figure 12 serves to show the effect of the data block length and the CIR length on the computational complexity of the HNN MLSE equalizer. Figure 12 shows the computational complexity for a more realistic scenario. Here the complexity of the BPSK HNN MLSE and the M-QAM HNN MLSE equalizer is shown for $N=1000, N=1500$ and $N=2000$, for $L=0$ to $L=1000$.

From Figure 12 it is clear that the computational complexity increases quadratically as the data block length linearly increases. It is quite significant that the complexity is nearly independent of the CIR length when the data block length is equal to or great then the CIR length, which is the case in practical communication systems. It should now be clear why the HNN MLSE equalizer is able to equalize signals in systems, employing BPSK or M-QAM modulation, with hundreds and possibly thousands of resolvable multipath elements.

The superior computational complexity of the HNN MLSE equalizer is obvious. Its low complexity makes it suitable for equalization of signals with CIR lengths that are beyond the capabilities of optimal equalizers like the Viterbi MLSE equalizer and the MAP equalizer, for which 


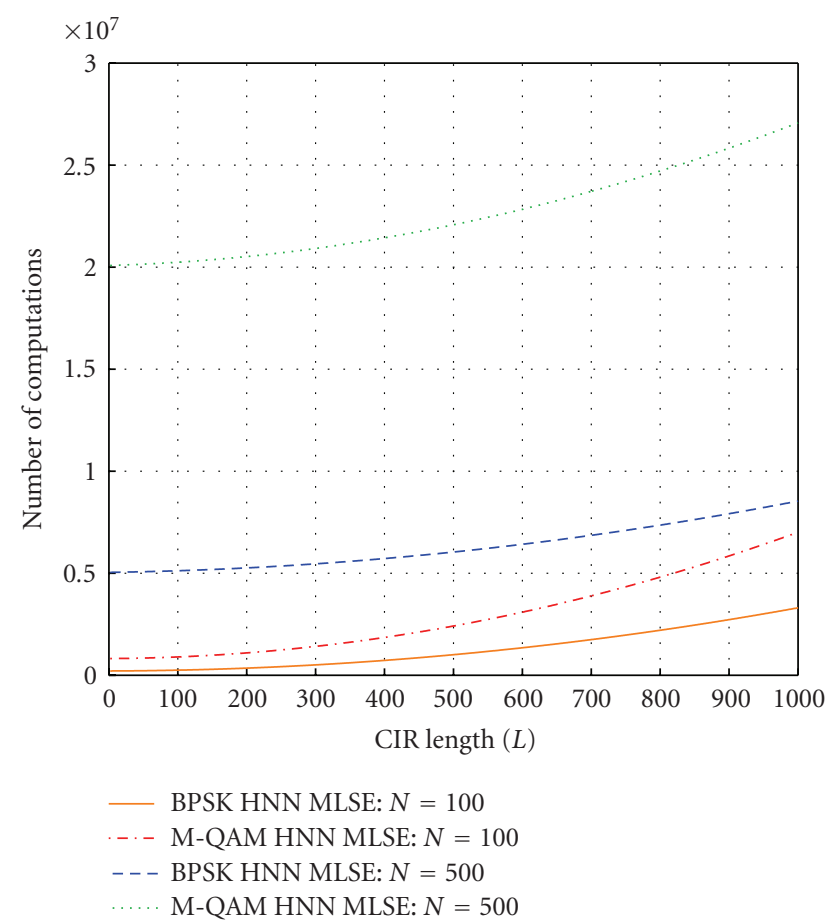

FIGURE 11: Computational complexity comparison for the HNN MLSE equalizers for different data block lengths.

the computational complexity increases exponentially with an increase in the channel memory (note that the computational complexity graphs of the Viterbi MLSE equalizer cannot be presented on the same scale as that of the HNN MLSE equalizer, as shown in Figure 10 through Figure 12), and it is also exponentially related to the number of symbols in the modulation alphabet. On the other hand, the computational complexity of the HNN MLSE equalizer is quadratically related to the data block length and almost independent of the CIR length for realistic scenarios. Also, the complexity of the HNN MLSE equalizer is independent of the modulation alphabet size for M-QAM systems, making it suitable for equalization in higher order M-QAM system with even moderate channel memory, where optimal equalizer cannot be applied.

\section{Simulation Results}

In this section, the HNN MLSE equalizer is evaluated. The low computational complexity of the HNN MLSE equalizer allows it to equalize signals in systems with extremely long memory, well beyond the capabilities of conventional optimal equalizers like the Viterbi MLSE and the MAP equalizers. The HNN MLSE equalizer is evaluated for long sparse and dense Rayleigh fading channels. It will be established that the HNN MLSE equalizer outperforms the MMSE equalizer in long fading channels and it will be shown that the performance of the HNN MLSE equalizer in sparse channels is better than its performance in equivalent dense channels, that is, longer channels with the same amount of nonzero CIR taps.

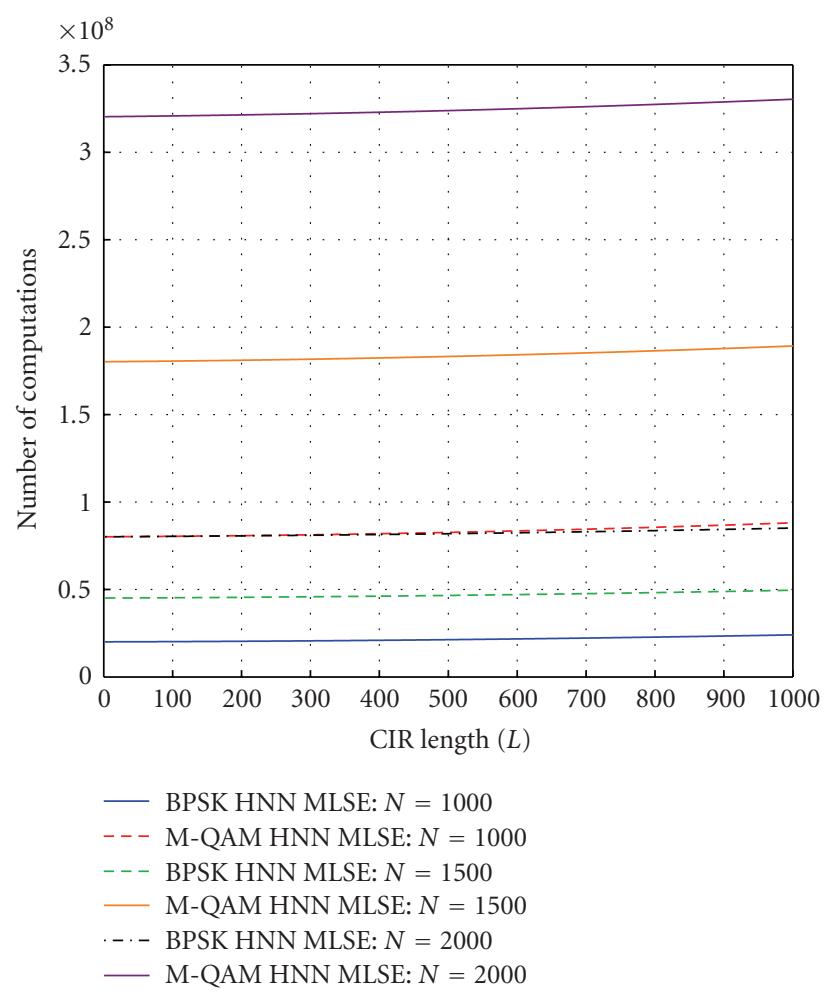

FIGURE 12: Computational complexity comparison for the HNN MLSE equalizers for realistic data block lengths.

The communication system is simulated for a GSM mobile fading environment, where the carrier frequency is $f_{c}=900 \mathrm{MHz}$, the symbol period is $T_{s}=3.7 \mu \mathrm{s}$ and the relative speed between the transmitter and receiver is $v=3 \mathrm{~km} / \mathrm{h}$. To simulate the fading effect on each tap, the Rayleigh fading simulator proposed in [33] is used to generate uncorrelated fading vectors. Least Squares (LS) channel estimation is used to determine an estimate for the $\mathrm{CIR}$, in order to include the effect of imperfect channel state information (CSI) in the simulation results. Where perfect CSI is assumed, however, the statistical average of each fading vector is used to construct the CIR vector for each received data block. In all simulations, the nominal CIR weights are chosen as $\mathbf{h}=\left\{h_{0} /\|\mathbf{h}\|, h_{1} /\|\mathbf{h}\|, \ldots, h_{L-1} /\|\mathbf{h}\|\right\}$ such that $\mathbf{h}^{T} \mathbf{h}=1$, where $L$ is the CIR length, and $\mathbf{h}$ is a column vector of length $L$, in order to normalize the energy in the channel. The normalized nominal taps are used to scale the uncorrelated fading vectors produced by the Rayleigh fading simulator. To simulated dense channels, all the nominal tap weights are chosen as 1 , after which the taps are normalized as explained. To simulated sparse channels, $K \%$ of the nominal taps weights are chosen as 1 (for sparse channels the nonzero taps are evenly spaced), while the rest are set to zero. Again the taps are normalized, but now only $K \%$ of $L$ taps are nonzero.

In order to compare the performance of the HNN MLSE equalizer in dense channels to its performance in sparse channels, an equivalent dense channel is used. An equivalent dense channel is a dense channel with an equal amount of nonzero taps as that of a sparse channel of any length. 


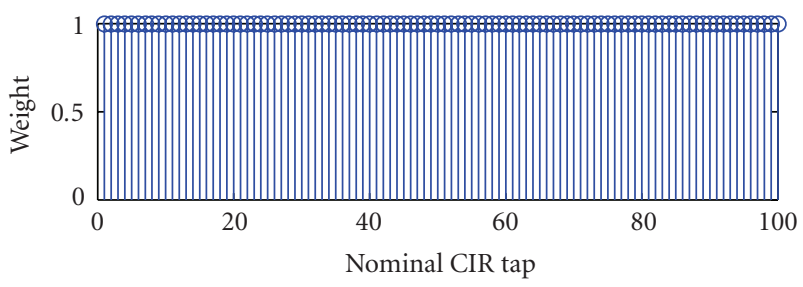

(a)

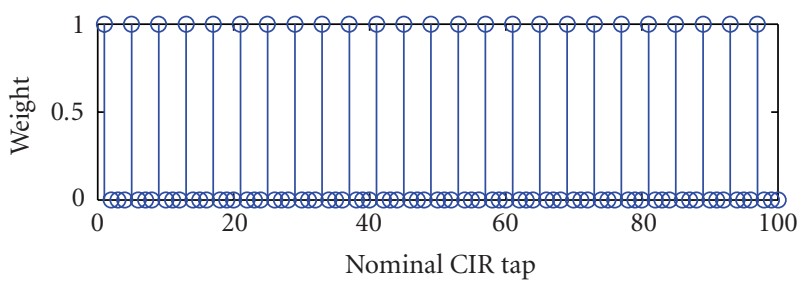

(c)

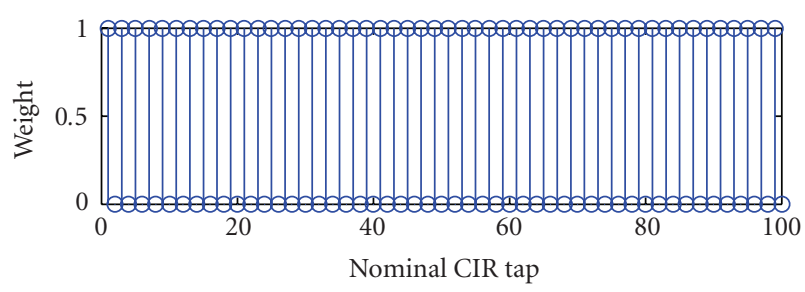

(b)

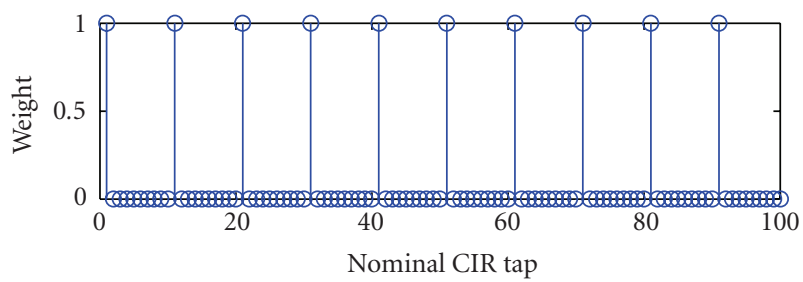

(d)

FIGURE 13: Normalized evenly spaced nominal CIR taps of sparse channels. (a) $K=100 \%$ (b) $K=50 \%$ (c) $K=25 \%$ (d) $K=10 \%$.

A sparse channel of length $L$, where only $K \%$ of the taps are nonzero, is compared to a dense channel of length $[L$. $K] / 100$, so that the number of nonzero taps in the sparse channel is equal to that in the equivalent dense channel. Figure 13 shows the evenly spaced normalized nominal CIR taps of sparse channels of length $L=100$, where $K=100 \%$ (a), $K=50 \%$ (b), $K=25 \%$ (c) and $K=10 \%$ (d), reducing the number of nonzero CIR taps to $K \%$ of the CIR length.

6.1. Performance in Dense Channels. The performance of the BPSK HNN MLSE equalizer and 16-QAM HNN MLSE equalizers is evaluated for long dense channels, for perfect CSI, with channel delays from $74 \mu$ s to $1.9 \mathrm{~ms}$ ( $L=10$ to $L=$ 500). The performance of the BPSK HNN MLSE equalizer is also compared to that of an MMSE equalizer for imperfect CSI.

Figure 14 shows the performance of the BPSK HNN MLSE equalizer for perfect CSI, where the uncoded data block length is $N=500$ and the CIR lengths range from $L=10$ to $L=500$, corresponding to channel delay spreads of $37 \mu$ s to $1.85 \mathrm{~ms}$. As the channel memory increases, the performance increases correspondingly, approaching unfaded matched filter performance as a result of the effective time diversity provided by the multipath channels. It is clear the BSPK HNN MLSE equalizer performs near-optimal, if not optimal, equalization. Note that for $L=500$, a Viterbi MLSE equalizer would require $M^{L-1}=2^{499}$ states in its trellis per transmitted symbol.

Figure 15 shows the performance of the 16-QAM HNN MLSE equalizer, where again perfect CSI and an uncoded data block length of $N=500$ are assumed. Here, the CIR length ranges from $L=25$ to $L=400$ which corresponds to channel delay spreads of $92.5 \mu \mathrm{s}$ to $1.48 \mathrm{~ms}$. Here, it is also clear that the performance approaches unfaded matched filter performance as the channel memory increases. Note that a Viterbi MLSE equalizer would require $M^{L-1}=16^{399}$ states in its trellis per transmitted symbol for $L=400$.

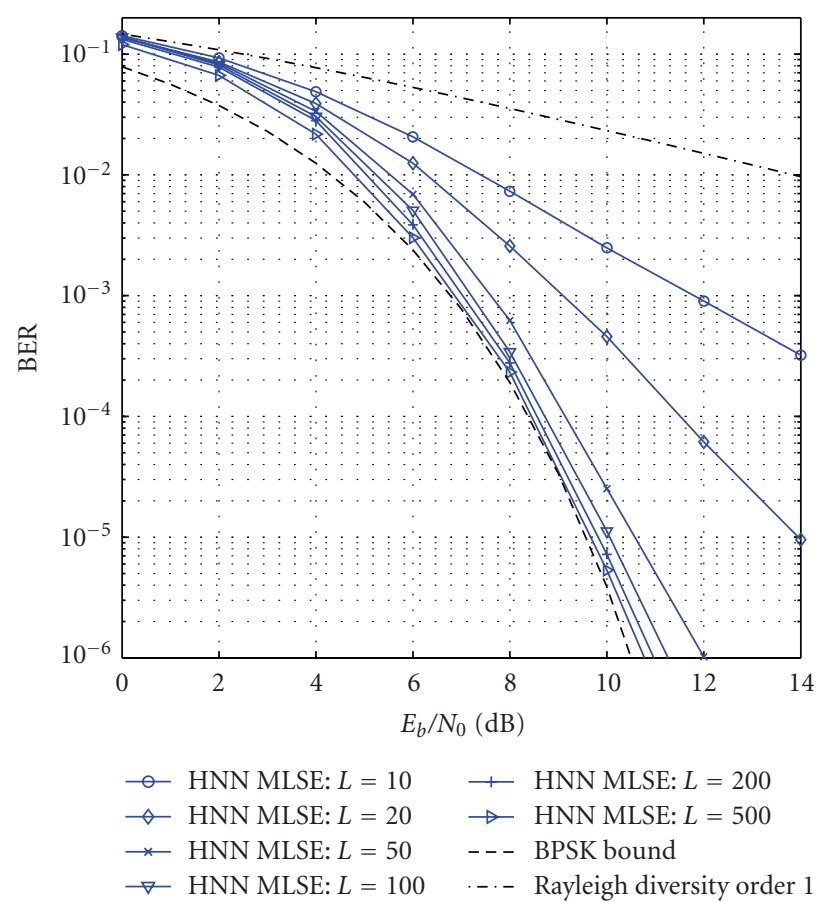

FIGURE 14: BPSK HNN MLSE equalizer performance in extremely long channels.

Figure 16 shows the performance of the BPSK HNN MLSE equalizer compared to that of an MMSE equalizer, for moderate to long channels, using channel estimation. It is assumed that $3 L$ training symbols are available for channel estimation per data block of length $N=450$. For the HNN MLSE equalizer the LS channel estimator is used to estimate the CIR using $3 L$ training symbols, and for the MMSE equalizer the channel is estimated as part of the filter coefficient optimization-an integral part of MMSE equalization - where the filter smoothing length is 


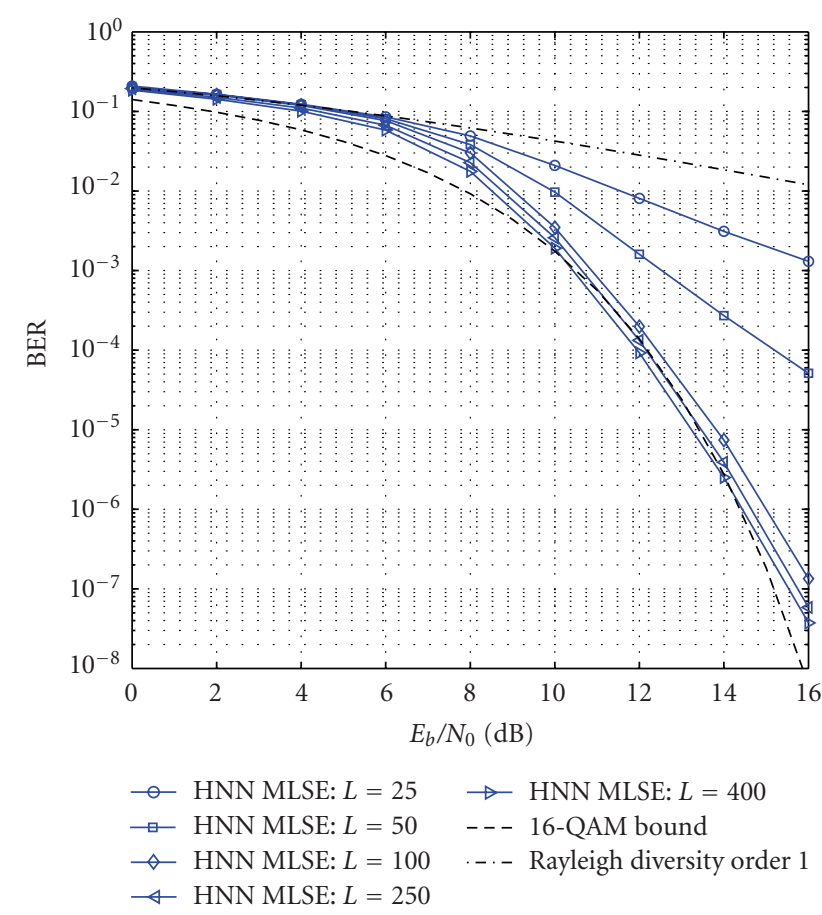

FIGURE 15: 16-QAM HNN MLSE equalizer performance in extremely long channels.

$3 L$. From Figure 16 it is clear that the HNN MLSE equalizer outperforms the MMSE equalizer for high $E_{b} / N_{0}$-levels and when the channel memory is large.

From these results it is clear that the HNN MLSE equalizer effectively equalizes the received signal and performs near-optimally when the channel memory is large, if perfect CSI is assumed.

6.2. Performance in Sparse Channels. The performance of the BSPK HNN MLSE equalizer, and 16-QAM HNN MLSE equalizers is evaluated for sparse channels, where their performance is compared to equivalent dense channels. The equalizer is simulated for various levels op sparsity, where $K$ indicates the percentage of nonzero CIR taps. The performance of the HNN MLSE equalizers in these sparse channels is compared to their performance in equivalent dense channels of length $L=10$ to $L=100$. Perfect CSI is assumed.

For the BPSK HNN MLSE equalizer an uncoded data block length of $N=200$ is used where the channel delay of the sparse channel is $0.74 \mathrm{~ms}$, corresponding to a CIR length of $L=100$. To simulate the BPSK equalizer $K$ is chosen as $K=10 \%, K=20 \%, K=40 \%, K=60 \%$, and $K=80 \%$, such that the number of nonzero taps in the CIR is $10,20,40$, 60 , and 80 , respectively. Figure 17 shows the performance of the BPSK HNN MLSE equalizer in a sparse channel of length $L=100$, compared to its performance in dense channels of length $L=10$ to $L=100$. It is clear from Figure 17 that the performance of the BPSK HNN MLSE equalizer in sparse is better compared to the corresponding equivalent dense channels. Because of the approximate independence of the computational complexity on the CIR length, the increase in complexity due to larger $L$ is negligible.

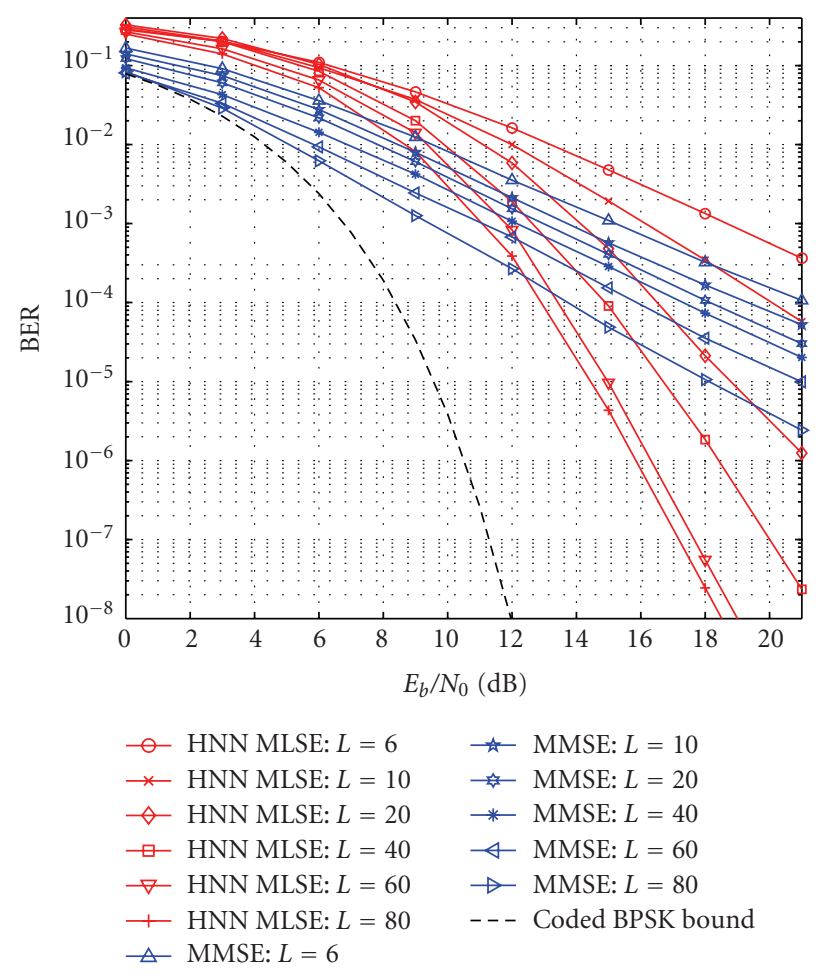

Figure 16: BPSK HNN MLSE and MMSE equalizer performance comparison.

The 16-QAM HNN MLSE equalizer is also simulated for sparse channels, where the uncoded block length is $N=200$ and the channel delay spread is $L=100$ which corresponds to $1.5 \mathrm{~ms}$. Here, $K$ is chosen as $K=25 \%$ and $K=40 \%$ such that the number of nonzero taps in the CIR is 25 and 40 , respectively. Figure 18 shows the performance of the 16QAM HNN MLSE equalizer in a sparse channel of length $L=100$, compared to its performance in dense channels of length $L=25$ to $L=100$. Here, again it is clear that the performance of the equalizer in sparse channels is better than its performance in the corresponding equivalent dense channels. Again, the complexity increase for equalization in sparse channels is negligible.

From the simulation results in Figures 17 and 18 it is clear that the HNN MLSE equalizer performs well in sparse channels compared to its performance in equivalent dense channels. Having an equal amount of nonzero nominal CIR taps, the performance increase in the sparse channels is not attributed to more diversity due to extra multipath, but rather to the higher level of interconnection between the neurons in the HNN. (Longer estimated CIRs will allow the connection matrix of the HNN to be more densely populated, increasing the level of interconnection between the neurons.) This allows the HNN MLSE equalizer to mitigate the effect of multipath more effectively to produce better performance in sparse channels than in their corresponding equivalent dense channels.

Figure 19 shows the performance of the BPSK HNN MLSE equalizer for various sparse channels, all with a fixed amount of nonzero nominal CIR taps such that the level 


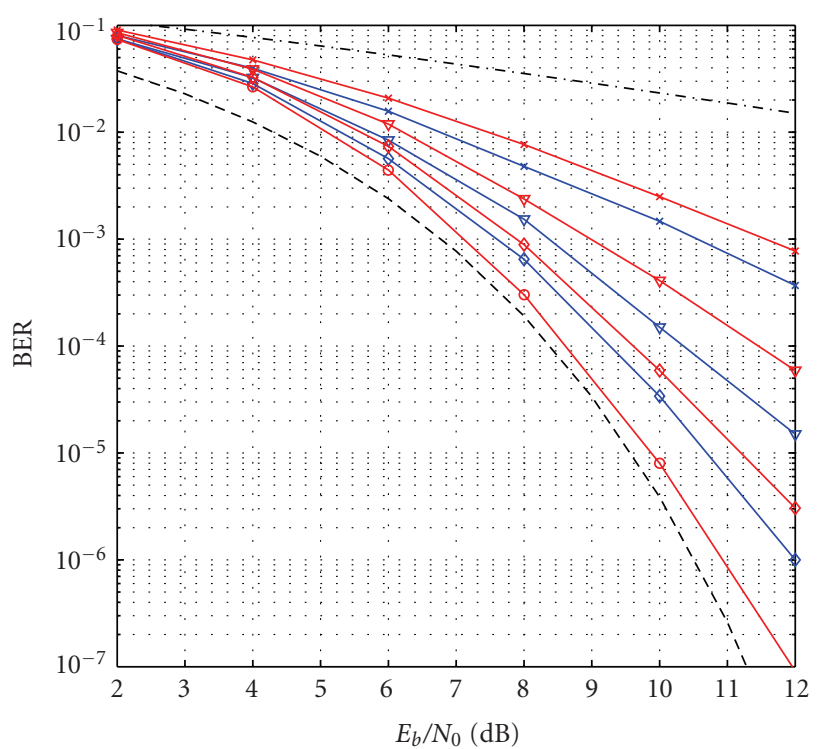

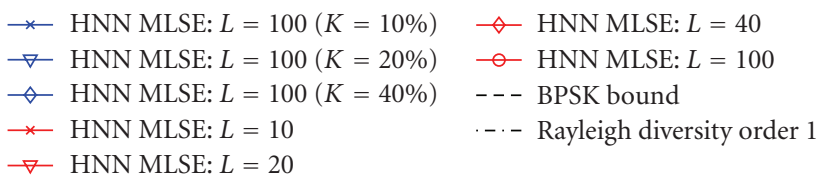

FIGURE 17: BPSK HNN MLSE equalizer performance in sparse channels.

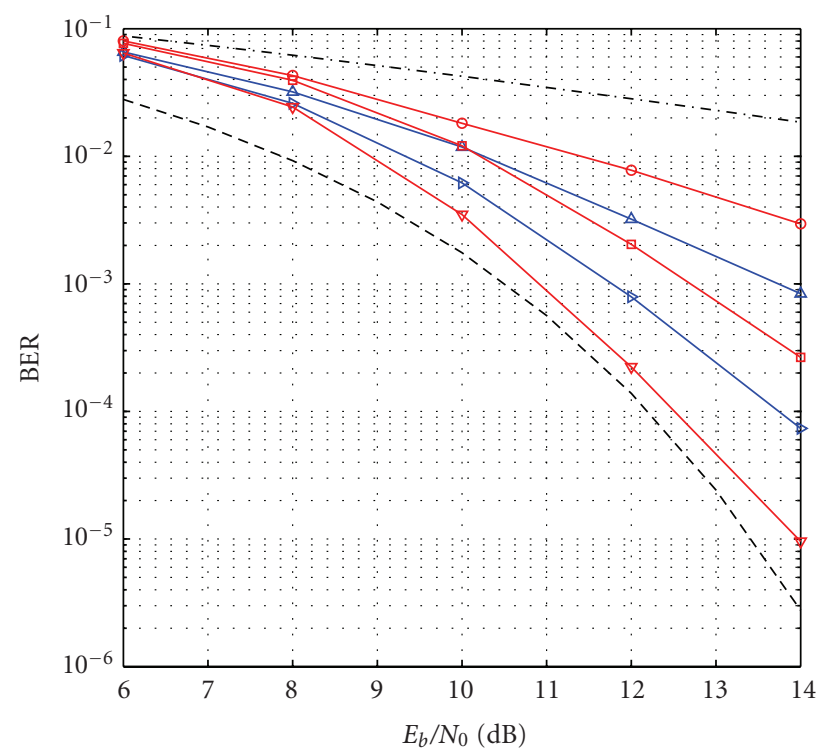

\footnotetext{
$\triangle$ HNN MLSE: $L=100(K=25 \%) \rightarrow \quad$ HNN MLSE: $L=100$

$\rightarrow$ HNN MLSE: $L=100(K=40 \%)---16$-QAM bound

$\rightarrow$ HNN MLSE: $L=25 \quad \cdots$ Rayleigh diversity order 1

$\rightarrow$ HNN MLSE: $L=40$
}

FIGURE 18: 16-QAM HNN MLSE equalizer performance in sparse channels.

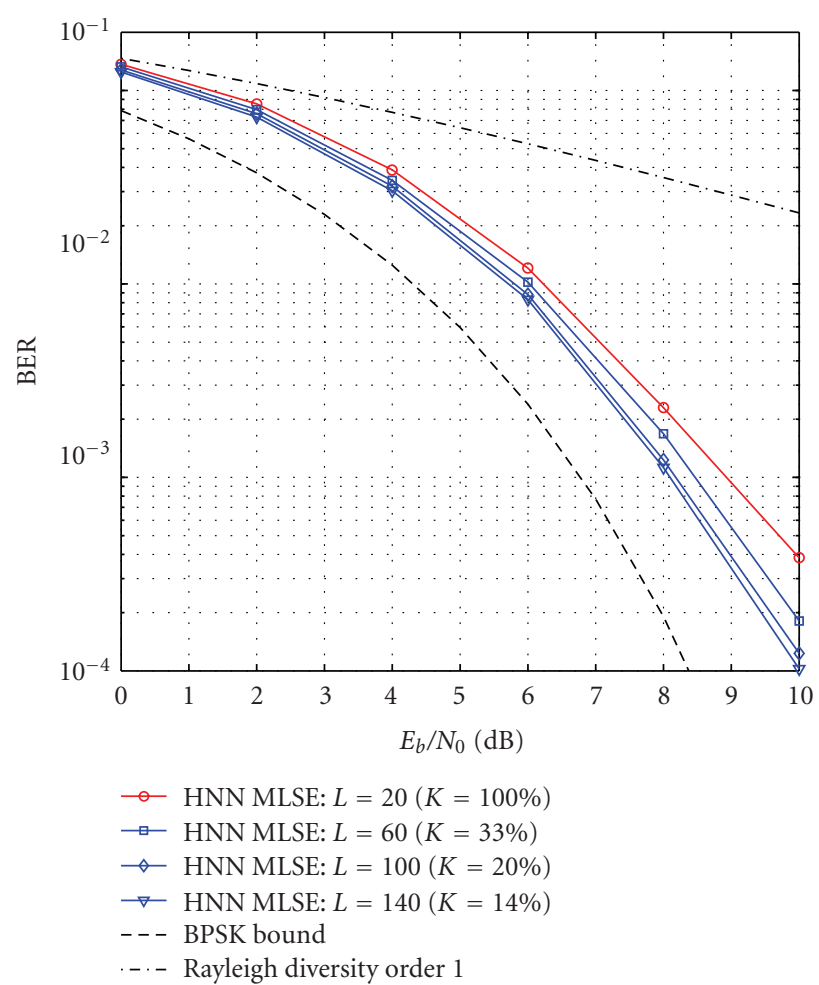

FIGURE 19: BPSK HNN MLSE performance in sparse channels with constant diversity.

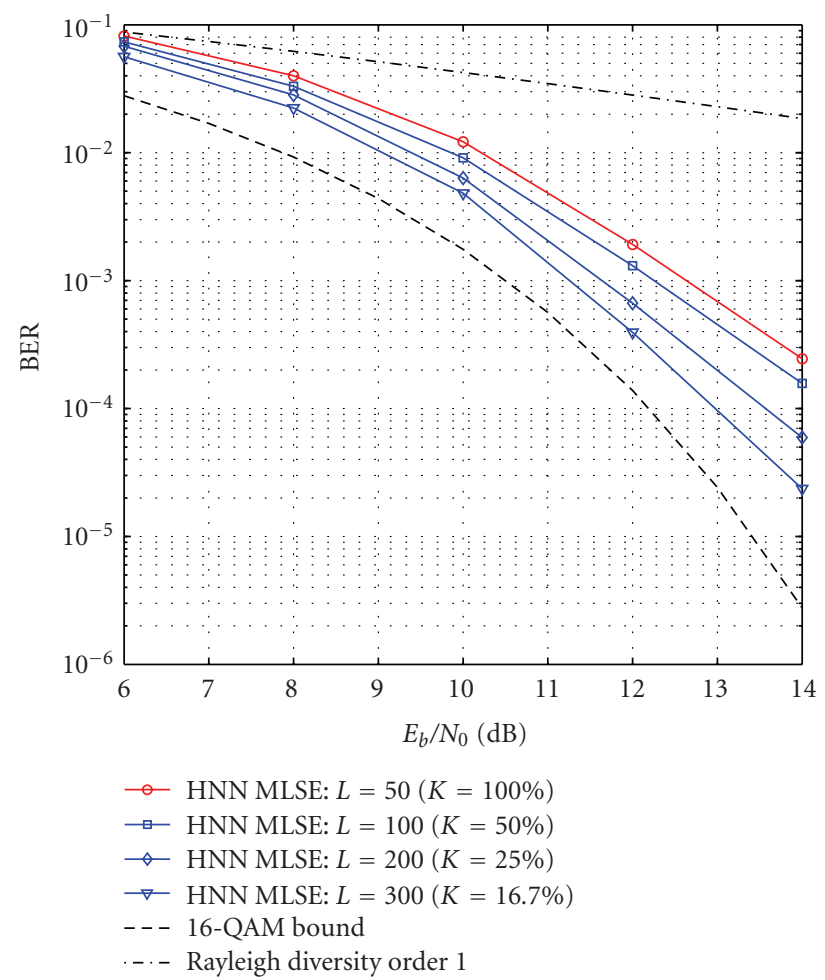

FIGURE 20: 16-QAM HNN MLSE performance in sparse channels with constant diversity. 
of diversity due to multipath remains constant. Using an uncoded data block length of $N=200$ and with $L$ such that $[L \cdot K] / 100=20$ for $K=100 \%, K=33 \%, K=$ $20 \%$ and $K=14 \%$ (corresponding to $L=20, L=60$, $L=100$ and $L=140$ ), it is clear that the performance increases as the CIR length $L$ increases, although the number of multipath channels remains unchanged for all cases. Also, Figure 20 shows the performance of the 16-QAM HNN MLSE equalizer. Using an uncoded data block length of $N=$ 400 and with $L$ such that $[L \cdot K] / 100=50$ for $K=100 \%$, $K=50 \%, K=25 \%$ and $K=16.7 \%$ (corresponding to $L=50, L=100, L=200$ and $L=300$ ), the performance increases with an increase in $L$.

From these results in is clear that the BER performance increases with an increase in $L$. The HNN MLSE equalizer thus exploits sparsity in communication channels by reducing the BER as the level of sparsity increases, given that the nonzero CIR taps remains constant.

\section{Conclusion}

In this paper, a low complexity MLSE equalizer was proposed for use in single-carrier M-QAM modulated systems with extremely long memory. The equalizer has computational complexity quadratic in the data block length and approximately independent of the channel memory length. An extensive computational complexity analysis was performed, and the superior computational complexity of the proposed equalizer was graphically presented. The $\mathrm{HNN}$ was used as the basis of this equalizer due to its low complexity optimization ability. It was also highlighted that the complexity of the equalizer for any single carrier M-QAM system is independent of the number of symbols in the modulation alphabet, allowing for equalization in 256-QAM systems with equal computational cost as for 4-QAM systems, which is not possible with conventional optimal equalizers like the VA and MAP.

When the equalizer was evaluated for extremely long channels for perfect CSI its performance matched unfaded AWGN performance, providing enough evidence to assume that the equalizer performs optimally for extremely long channels. It is therefore assumed that the performance of the equalizer approaches optimality as the connection matrix of the HNN is populated. It was also shown that the HNN MLSE equalizer outperforms an MMSE equalizer at high $E_{b} / N_{0}$ values.

The HNN MLSE equalizer was evaluated for sparse channels and it was shown that its performance was better compared to its performance in equivalent dense channels, with a negligible increase in computational complexity. It was also shown how the equalizer exploits channel sparsity. The HNN MLSE equalizer is therefore very attractive for equalization in sparse channels, due to its low complexity and good performance.

With its low complexity equalization ability, the HNN MLSE equalizer can find application in systems with extremely long memory lengths, where conventional optimal equalizers cannot be applied.

\section{References}

[1] G. D. Forney Jr., "Maximum likelihood sequence estimation of digital sequences in the presence of intersymbol interference," IEEE Transactions on Information Theory, vol. 18, no. 3, pp. 363-378, 1972.

[2] A. D. Viterbi, "Error bounds for convolutional codes and an asymptotically optimum decoding algorithm," IEEE Transactions on Information Theory, vol. 13, no. 1, pp. 260-269, 1967.

[3] L. R. Bahl, J. Cocke, F. Jelinek, and J. Raviv, "Optimal decoding of linear codes for minimizing symbol error rate," IEEE Transactions on Information Theory, vol. 20, no. 2, pp. 284287, 1974.

[4] J. G. Proakis, Digital Communications, McGraw-Hill, New York, NY, USA, 4th edition, 2001.

[5] A. Duel-Hallen and C. Heegard, "Delayed decision-feedback sequence estimation," IEEE Transactions on Communications, vol. 37, no. 5, pp. 428-436, 1989.

[6] W. U. Lee and F. S. Hill Jr., "A maximum likelihood sequence estimator with decision feedback equalizer," IEEE Transactions on Communications, vol. 25, no. 9, pp. 971-979, 1977.

[7] W. H. Gerstacker and R. Schober, "Equalization concepts for EDGE," IEEE Transactions on Wireless Communications, vol. 1, no. 1, pp. 190-199, 2002.

[8] A. Goldsmith, Wireless Communications, Cambridge University Press, Cambridge, UK, 2005.

[9] J. Terry and J. Heiskala, OFDM Wireless LANs: A Theoretical and Practical Guide, Sams, Indianapolis, Ind, USA, 2001.

[10] R. R. Lopes and J. R. Barry, "The soft-feedback equalizer for turbo equalization of highly dispersive channels," IEEE Transactions on Communications, vol. 54, no. 5, pp. 783-788, 2006.

[11] M. Stojanovic, L. Freitag, and M. Johnson, "Channelestimation-based adaptive equalization of underwater acoustic signals," Oceans, vol. 2, pp. 985-990, 1999.

[12] M. Zimmermann and K. Dostert, "A multipath model for the powerline channel," IEEE Transactions on Communications, vol. 50, no. 4, pp. 553-559, 2002.

[13] J. W. M. Bergmans, Digital Baseband Transmission and Recording, Springer, New York, NY, USA, 1996.

[14] H. C. Myburgh and J. C. Olivier, "Low complexity iterative MLSE equalization of M-QAM signals in extremely long rayliegh fading channels," in Proceedings of the European International Science Fiction Convention (EUROCON '09), pp. 1632-1637, 2009.

[15] J. J. Hopfield and D. W. Tank, "Neural computation of decisions in optimization problems," Biological Cybernetics, vol. 52, no. 3, pp. 141-152, 1985.

[16] H. H. Zeng, L. Ye, and J. H. Winters, "Improved spatialtemporal equalization for EDGE: a fast selective-direction MMSE timing recovery algorithm and two-stage soft-output equalizer," IEEE Transactions on Communications, vol. 49, no. 12, pp. 2124-2134, 2001.

[17] X. Dayong, X. Yang, and D. Haifeng, "An improved algorithm of MMSE multiuser detection for CDMA systems," in Proceedings of the International Symposium on Communications and Information Technologies (ISCIT '05), vol. 1, pp. 552-555, 2005.

[18] H. Zhou and S. Zhou, "Improved adaptive MMSE detector for downlink multi-cell MIMO signals," in Proceedings of the 60th IEEE Vehicular Technology Conference (VTC '04), pp. 37333737, September 2004.

[19] N. Czink, G. Matz, D. Seethaler, and F. Hlawatsch, "Improved MMSE estimation of correlated MIMO channels using 
a structured correlation estimator," in Proceedings of the IEEE Workshop on Signal Processing Advances in Wireless Communications (SPAWC '05), pp. 595-599, 2005.

[20] J.-H. Park, Y. Whang, and K. S. Kim, "Low complexity MMSESIC equalizer employing time-domain recursion for OFDM systems," IEEE Signal Processing Letters, vol. 15, pp. 633-636, 2008.

[21] Z. Rong and L. Guangqiu, "Low complexity code multiplexed pilot aided adaptive MMSE equalizerfor CDMA systems," in Proceedings of the International Conference on Wireless Communications, Networking and Mobile Computing, pp. 1-4, 2008.

[22] S. H. Bang, B. J. Sheu, and R. C.-H. Chang, "Maximum likelihood sequence estimation of communication signals by a Hopfield neural network," in Proceedings of the IEEE International Conference on Neural Networks, vol. 5, pp. 33693374, July 1994.

[23] S. H. Bang and B. J. Sheu, "A neural network for detection of signals in communication," IEEE Transactions on Circuits and Systems I, vol. 43, no. 8, pp. 644-655, 1996.

[24] J. D. Provence, "Neural network implementation for an adaptive maximum-likelihood receiver," in Proceedings of the IEEE International Symposium on Circuits and Systems, vol. 3, pp. 2381-2385, June 1988.

[25] D. C. Chen, B. J. Sheu, and E. Y. Chou, "A neural network communication equalizer with optimized solution capability," in Proceedings of the IEEE International Conference on Neural Networks, vol. 4, pp. 1957-1962, June 1996.

[26] J. J. Hopfield, "Artificial neural networks," IEEE Circuits and Devices Magazine, vol. 4, no. 5, pp. 3-10, 1988.

[27] J. C. Olivier, "Essential Digital Communication Theory," ESF320 Lecture notes, 2007.

[28] S. Russell and P. Norvig, Artificial Intelligence: AModern Approach, Prentice-Hall, Upper Saddle River, NJ, USA, 2nd edition, 2003.

[29] S. H. Bang, O. T.-C. Chen, J. C.-F. Chang, and B. J. Sheu, "Paralleled hardware annealing in multilevel Hopfield neural networks for optimal solutions," IEEE Transactions on Circuits and Systems II, vol. 42, no. 1, pp. 46-49, 1995.

[30] S. H. Bang, B. J. Sheu, and J. C.-F. Chang, "Search of optimal solutions in multi-level neural networks," in Proceedings of the IEEE International Symposium on Circuits and Systems (ISCAS '94), vol. 6, pp. 423-426, June 1994.

[31] U. Halici, "Artificial Neural Networks," EE 543 Lecture notes, 2004.

[32] B. Farhang-Boroujeny, H. Zhu, and Z. Shi, "Markov chain Monte Carlo algorithms for CDMA and MIMO communication systems," IEEE Transactions on Signal Processing, vol. 54, no. 5, pp. 1896-1909, 2006.

[33] Y. R. Zheng and C. Xiao, "Improved models for the generation of multiple uncorrelated Rayleigh fading waveforms," IEEE Communications Letters, vol. 6, no. 6, pp. 256-258, 2002. 